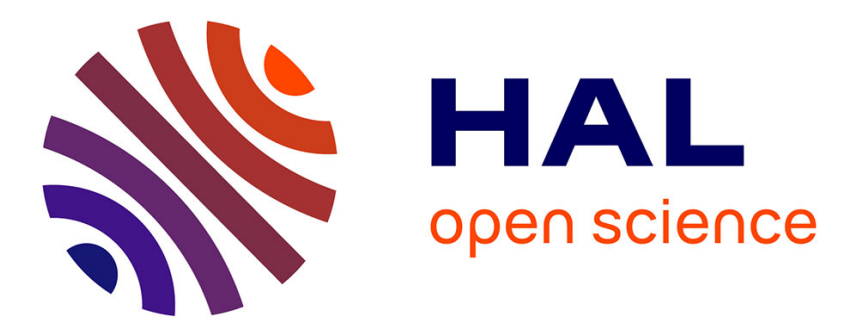

\title{
Quand les rhinocéros prennent la parole: le gab et la question de la parole efficace dans Le Voyage de Charlemagne à Jérusalem et à Constantinople
}

\author{
Éléonore Andrieu
}

\section{To cite this version:}

Éléonore Andrieu. Quand les rhinocéros prennent la parole: le gab et la question de la parole efficace dans Le Voyage de Charlemagne à Jérusalem et à Constantinople. N. Bériou, J.-P. Boudet, I. RosierCatach dir. Le pouvoir des mots au Moyen Âge, Brepols, p. 71-106, 2014. hal-02148045

\section{HAL Id: hal-02148045 \\ https://hal.science/hal-02148045}

Submitted on 5 Jun 2019

HAL is a multi-disciplinary open access archive for the deposit and dissemination of scientific research documents, whether they are published or not. The documents may come from teaching and research institutions in France or abroad, or from public or private research centers.
L'archive ouverte pluridisciplinaire HAL, est destinée au dépôt et à la diffusion de documents scientifiques de niveau recherche, publiés ou non, émanant des établissements d'enseignement et de recherche français ou étrangers, des laboratoires publics ou privés. 
ELEONORE ANDRIEU*

\title{
QUAND LES RHINOCEROS PRENNENT LA PAROLE : LE GAB ET
}

\section{LA QUESTION DE LA PAROLE EFFICACE DANS LE VOYAGE DE}

\author{
CHARLEMAGNE A JERUSALEM ET A CONSTANTINOPLE
}

\section{LA PAROLE ET LES DEUX GENRES D’HOMMES}

La Vie latine d'Isarn, abbé de Saint-Victor de Marseille, raconte comment, à la suite du pillage du monastère de Lérins par des païens, certains frères furent emmenés, captifs, en Espagne. Le monastère demanda l'aide de l'abbaye de Saint-Victor : l'abbé Isarn décida de se rendre en personne en Catalogne, alors même qu'une grave maladie le tenait désormais presque constamment alité. Une fois parvenu au prieuré victorin de Saint-Michel del Fai, il y reçut le comte de Barcelone, son épouse, et leur allié Gombaud de Besora ${ }^{1}$. Voici le récit de cette scène :

La rumeur de son arrivée étant parvenue jusqu'à eux, le consul de Barcelone Raimond Bérenger et sa femme Elisabeth, ainsi que Gombaud, homme de rang consulaire, c'est-à-dire les plus grands princes de toute la région, se hâtent vers ce lieu pour s'offrir eux-mêmes et leurs biens, dévoilent leurs péchés et le prient de se faire leur défenseur auprès de Dieu. Et Isarn, les édifiant grandement par les paroles de l'Ecriture, leur imposa aussitôt comme œuvre de pénitence de restituer au Seigneur Dieu les moines captifs, par tous les moyens possibles et appropriés, aussi bien par les menaces de leur puissance que par les forces de la persuasion, par l'argent et par la supplication, et de restaurer, pour l'honneur du Seigneur, le monastère de Lérins jadis illustre et maintenant injustement détruit par des peuples impies. Et s'ils le faisaient, il leur promettait qu'ils obtiendraient facilement du Seigneur la rémission de leurs péchés, si du moins ils se corrigeaient, et de prier pour eux de tout son cœur. Alors, inspirés par Celui qui arme les rhinocéros d'une corne de puissance de ce genre pour leur permettre de dompter l'esprit des barbares, et qui par de tels verrous place des limites à la mer amère et turbulente des païens, ils s'avancent, prêts à accomplir la totalité de son vœu².

\footnotetext{
* Université Michel de Montaigne - Bordeaux III / EA 4195 TELEM / elandrieu @ wanadoo.fr

${ }^{1}$ Pour la bibliographie concernant ce passage et ses acteurs, voir la Vie d'Isarn, abbé de Saint-Victor de Marseille (XI siècle), présentation, édition, traduction et notes, C. Caby, J.-F. Cottier, R. M. Dessí, M. Lauwers, J.-P. Weiss, M. Zerner, Paris, 2010 (Les classiques de l'histoire au Moyen Age), p. 78-96.

${ }^{2}$ Ibidem, XXXI, p. 88-91. Voici le texte latin : "Tunc adventus eius rumore respersi Barcinonae consul Raimundus Berengarii et uxor eius Elisabeth et Gumbaldus vir consularis, illuc se suaque offerentes, principes scilicet maximi totius regionis, accederant, peccata propria detegunt, patronumque illum sibi apud Deum fieri deprecantur. Quos ille multum sermonibus divinis aedificans, id eis protinus paenitentiae munus iniunxit ut, quibuscunque iuste possent modis, tam potentiae minis quam ingenii viribus, et censu et prece captivos monachos Domino Deo restituerent, ac nobile quondam Linirense monasterium, nunc inique ab impiis destructum gentibus, ad honorem Domini restaurarent. Quod si facerent, remissionem illis peccatorum, si se corrigerent, a Domino facile pollicetur, seque pro eis totis visceribus precaturum. Tunc aspirante illo, qui rinocerotas huiusmodi cornu potentiae ad edomandos barbarorum animos armat, et per tales vectes amarissimo turbulentoque gentium mari terminos ponit, ad omne votum eius sese promptissimos offerunt ».
} 
Ce texte de la seconde moitié du $\mathrm{XI}^{\mathrm{e}}$ siècle met en scène un «nouveau paradigme social, caractéristique des réformateurs grégoriens ${ }^{3} »:$ d'un côté, les « grands princes » de la terre. Ce sont des rhinocéros (armés de la «corne de puissance », argent ou forces de ce monde), ou encore les «verrous de la mer amère ». De l'autre, le saint abbé : Isarn dispose de la parole de Dieu ${ }^{4}$ et il est, pour les laïcs qui accourent vers lui, le médiateur exclusif dans l'accès aux choses spirituelles. La force de ses mots, à la fois «édification », « ordre », «promesse », «prière » et «vœu », rend opératoire la défense matérielle de l'Eglise par les aristocrates laïcs : l'abbé modèle ainsi l'action des rhinocéros armés selon les contours de son vжu et en fait une œuvre de pénitence.

Le portrait du grand aristocrate laïc en rhinocéros, particulièrement exploité dans l'hagiographie clunisienne, se fonde sur Iob $39,9-10^{5}$ et revêt un sens précis ${ }^{6}$ : jugulé par l'Eglise (les princes «s'offrent, eux-mêmes et leurs biens »), l'animal « met sa puissance à son service et peut être utilisé par elle pour piétiner ses ennemis » alors que, « hors de l'Eglise, le rhinocéros se déchaîne contre elle ${ }^{7} \gg$. Le portrait de l'homme de Dieu en homo spiritualis évoque quant à lui I Cor. 2-15, texte dont Yves Sassier rappelle qu'il est l' ' un des fondements scripturaires de la grande revendication de l'Eglise des temps grégoriens : affirmer la supériorité intrinsèque du spirituel sur le temporel » et imposer «l'existence d'une hiérarchie inscrite dans la création justifiant pour toujours l'autorité des $\operatorname{clercs}^{8} »$. Comme le précise Dominique Iogna-Prat, les «mises en ordres » de la société proposées par les réformateurs consistent bien, à partir de la période grégorienne, à « définir la part des laïcs comme un reste ${ }^{9}$ » et à garantir par de nouveaux « devoirs » la supériorité éthique et sociale des ecclésiastiques. Ces derniers s'assurent notamment la possession exclusive du sacré et le pouvoir de médiation sacramentelle.

Or les problématiques de la parole efficace - qui accomplit ce qu'elle profère dans les sacrements et dans l'Histoire puisqu'elle donne aux hommes le sens de leurs actes - sont pleinement mobilisées dans les nouvelles propositions de «mises en ordres » de la société et dans les nouvelles représentations des « hommes spirituels » et de leur pouvoir, pouvoir matériel et idéel, pouvoir sur le monde et sur le salut des hommes ${ }^{10}$. Le sacrement de la consécration eucharistique n'est-il pas par exemple «celui dont la répétition scelle originellement et renouvelle quotidiennement l'unité de l'Eglise chrétienne ${ }^{11}$ ", ainsi que le rappelle Irène Rosier-Catach, alors même que son sens se modifie profondément à la

\footnotetext{
${ }^{3}$ Ibidem, p. 131.

${ }^{4}$ Le texte précise d'ailleurs à propos de cette parole divine qu'il « ne l'a pas dans son esprit » (chap. VII, p. 20-21), mais «devant ses yeux » ou encore «inscrite en son cœur» (chap. IV, p. 15-16), comme le font remarquer les éditeurs du texte : Vie d'Isarn, « Notes complémentaires », VII- «Usages de l'Ecriture sainte et modèles scripturaires dans la Vie d'Isarn », p. 153-154.

${ }^{5}$ Cfr les Moralia de Grégoire le Grand : 31, 1-7.

${ }^{6} \mathrm{P}$. Buc, L'ambiguïté du Livre. Prince, pouvoir et peuple dans les commentaires de la Bible au Moyen Age, Paris, 1994 ; B. Rosenwein, Rhinoceros Bound : Cluny in the Tenth Century, Philadelphia, 1982.

${ }^{7}$ Cf. les commentaires de la Vie d'Isarn, p. 89, n. 157, et les « Notes complémentaires », p. 102-171:p. 130 et p. $154-158$.

${ }^{8}$ Y. Sassier, «L'Histoire du monastère de Vézelay de Hugues de Poitiers, reflet des tensions idéologiques du XII ${ }^{\mathrm{e}}$ siècle », Structures du pouvoir, royauté et Res Publica (France, IX $X^{e}$-XII siècle), Rouen, 2004, p. 163175, p. 164. Je renvoie ici, sans aucune prétention d'exhaustivité, aux travaux suivants : G. Constable, The Reformation of the Twelfth Century, Cambridge, 1996 ; D. Iogna-Prat, Ordonner et exclure. Cluny et la société chrétienne face à l'hérésie, au judaïsme et à l'islam (1000-1150), Paris, 1998 ; G. Tellenbach, The Church in Western Europe from the Tenth to the Early Twelfth Century, Cambridge, 1993 (trad. anglaise).

${ }^{9}$ D. Iogna-Prat, Ordonner et exclure, p. 27.

${ }^{10}$ D. Iogna-Prat, Ordonner et exclure, p. $26 s q$.

${ }^{11}$ La parole efficace. Signe, rituel, sacré, Paris, 2004, p. 23.
} 
période grégorienne, donnant aux ministres de l'Eglise un rôle inédit et très discuté dans l'efficacité du sacrement ${ }^{12}$ ? Ainsi,

Whereas early Christianity looked to holy men and early medieval society turned to saints to effect the connexion between God and humankind through prayers of intercession, a different order was now emerging. It was embedded in procedures and mediating practices, in a neatly defined mystery, rather than in the inspiration of charismatic and exemplary figures. Now the unifying grace was being claimed and disposed of through strong sacramental routines which orientated Christian life on earth. [...] The priest was endowed with the power to effect a singular transformation in the world, one which was vital and necessary, so the claim of mediation was developed in the twelfth century into a robust theology of sacramentality ${ }^{13}$.

${ }^{12}$ H. de Lubac, Corpus mysticum. L'eucharistie et l'Eglise au Moyen Age, Paris, 1941 ; M. Rubin, Corpus Christi. The Eucharist in Late Medieval Culture, Cambridge, 1991, surtout p. 14 sq, pour les débats entre conceptions figuratives et conceptions réalistes de l'eucharistie. Cfr plus particulièrement, sur le problème connexe du pouvoir d'intercession inédit conféré au ministre de Dieu dans l'Eglise, I. RosierCatach, «Les sacrements comme signes qui font ce qu'ils signifient: signe efficace $v s$. efficacité symbolique », Versus, 102 (sept.-déc. 2006), surtout p. 172-174 sur « le rituel et le consensus » et La parole efficace, surtout p. 132-134 : en fait, le problème, analysé par l'auteur dans les textes théologiques, selon lequel «Dieu ne lie pas son pouvoir aux sacrements» est tout à fait central en contexte grégorien de construction d'une Eglise hiérarchique, institutionnelle et médiatrice. Comment en effet penser, par rapport à la grâce divine, le rôle de l'institution ecclésiale, du ministre de l'Eglise et de fait, du sacrement, avec les objets qu'il mobilise et les paroles qu'il nécessite ? Comment maintenir et affirmer le rôle central du prêtre dans la diffusion de la grâce à travers le sacrement, mais aussi l'équilibrer et le nuancer par rapport au pouvoir de l'Eglise et à la validité du sacrement en soi et pour soi, par rapport aussi à l'autonomie de la grâce divine? Quand les textes des théologiens examinés par I. Rosier-Catach (mais aussi les textes hagiographiques et le corpus juridique) discutent de la part de l'intervention humaine dans le sacrement à partir notamment de l'idée selon laquelle Dieu a « librement déterminé sa volonté à lier la confération de la grâce à ce moyen particulier » (paroles et matières du sacrement dans l'Eglise), ils posent aussi la question de « la part de pouvoir délégué à ces médiations, et [des] modalités selon lesquelles celles-ci agissent » (p. 134). D'où les débats et polémiques de la période grégorienne au sujet de l'autonomie du sacrement, question qui rejoint celle de l'autonomie des miracles défendue par exemple par Pierre Damien, comme l'a montré J.-M. Sansterre. Pierre Damien ainsi, dans une lettre adressée à l'archevêque Henri de Ravenne sur la validité des ordinations simoniaques, déclare : «il n'est pas étonnant que Dieu tout-puissant permette que l'office sacerdotal soit propagé dans son Eglise par de mauvais ministres, puisqu'il montre fréquemment par ceux-là même des miracles, évidemment non par le mérite d'une vie religieuse, mais en raison du ministère sacerdotal qu'ils ont reçu » (cité et traduit dans «L'autonomie du miracle chez Pierre Damien », J.-M. Sansterre, N. Stalmans, "Scribere sanctorum gesta». Recueil d'études d'hagiographie médiévale offert à Guy Philippart, éd. E. Renart, M. Trigalet, X. Hermand, P. Bertrand, Turnhout, 2005, p. 711-715, p. 712). Les controverses sont nombreuses, même au sein de l'Eglise grégorienne : I. Rosier-Catach rappelle ainsi que, pour les sacrements, la question de l'intention du ministre reste centrale, alors même qu'à côté des conditions de validité des sacrements que sont le « rôle ministériel du prêtre » et « la nécessité du respect du rite sous tous ses aspects », la « condition sur l'intention » «s'articule difficilement avec les précédentes, qui semblent en elles-mêmes nécessaires et suffisantes »(p. 266)... Telles que les analyse avec précision I. Rosier-Catach, les débat sur la nature exacte de la causalité à l'œuvre depuis Dieu jusqu'à l'effet à travers son ministre, son ministère, son institution, les règles de mise en scène du sacrement et les matières qu'il mobilise éventuellement, apparaissent pleinement liés à la construction de l'Eglise grégorienne, et de la société qu'elle entend définir et "inclure » après s'être hiérarchisée strictement: ce projet grégorien ne pouvait que ménager, d'une manière ou d'une autre, la part du ministre et celle de l'institution dans le dévoilement, voulu par Dieu, de sa grâce qui, dans tous les cas, reste la " cause efficiente dernière de l'effet du sacrement » (I. Rosier-Catach, «Le pouvoir des mots. Remarques sur la notion de causalité naturelle », Revue de synthèse, tome $129,6^{\mathrm{e}}$ série, $\mathrm{n}^{\circ} 4$, (2008), p. 611-616, p. 614).

${ }^{13}$ M. Rubin, Corpus Christi, p. 13. 
Ce même motif de la parole participe pleinement aux nouvelles configurations du grand aristocrate laïc, d'abord par contrepoint. On trouve dans la Vie de Geoffroy du Chalard, vers 1125 , cette déclaration prêtée par le saint lui-même à son prédécesseur dans les solitudes limousines :

«Je n'ai aucun pouvoir, car je suis un laïc et je ne suis pas expert en paroles ${ }^{14}$. »

La Vie d'Isarn offre de même un exemple intéressant de la manière dont le motif de la parole peut être utilisé : il y est entrelacé avec celui de la fonction guerrière dévolue aux grands aristocrates laïcs. Tout d'abord, ce texte les prive d'une parole efficace et les cantonne au rôle de défenseurs contractuels (par les armes, l'argent, la rhétorique de la persuasion) de l'Eglise terrestre. De fait, la fonction de parole et la fonction guerrière sont hiérarchisées en valeur : la parole efficace est de nature spirituelle et s'exerce au-delà de l'Histoire, tandis que le pouvoir des armes est temporel, provisoire et séculier. Mais cette hiérarchisation est dynamique : elle ne se contente pas d'opposer les deux fonctions, ou les deux genres d'hommes. Elle donne à la fonction de parole une vertu englobante par rapport à l'autre fonction, qu'elle récapitule ${ }^{15}$ : le texte présente explicitement le pouvoir des mots du saint auprès de Dieu comme l'arme utilisée dans une autre " défense » bien supérieure en valeur à celle qu'assurent les armes des rhinocéros dans l'Histoire. Inversement, les actes des grands princes sont dotés de valeur par la parole de prédication qui les fait, selon le vocabulaire de la conversion monastique ${ }^{16}$, «s'offrir » au désir du saint et à la volonté de Dieu : l'action guerrière procède donc de la parole de prédication et d'exhortation qui en est la «cause» au sens où elle est chargée de révéler le dessein divin. Cette structuration hiérarchique du motif des armes et du motif de la parole efficace implique que les armes, attribut central du laïc-rhinocéros, «jouissent d'une forme propre, quoiqu'humiliée, de reconnaissance ${ }^{17} »$, à la condition nécessaire d'être la forme même donnée au «vœu» de l'homme spirituel. Il est permis de rappeler qu'en accord avec ce modèle, la fonction guerrière est représentée dans nombre de textes de la période ${ }^{18}$ comme

\footnotetext{
14 «Ego obsistendi nullam potestatem habeo, quod laicus sum et imperitus sermone » (Vie de Geoffroy du Chalard, éd. A. Bosvieux, dans Mémoires de la Société des Sciences naturelles et archéologiques de la Creuse, III, 1862, p. 75-120, p. 82). La traduction est reprise de celle de M. Aubrun : Saints ermites en Limousin au XII siècle, traduction et présentation par M. Aubrun, Turnhout, 2009, p. 57-100, p. 65.

${ }^{15}$ L. Dumont, Homo hierarchicus ; le système des castes et ses implications, Paris, 1979.

${ }^{16}$ Vie d'Isarn, « Notes complémentaires », IV- « Récits 'antiseigneuriaux' et conversions au monachisme dans la Vie d'Isarn », p. 126-131, p. 130.

${ }^{17}$ Selon la formule de J. Lecointe : L'Idéal et la différence. La perception de la personnalité littéraire à la Renaissance, Genève, 1993, p. 113.

${ }^{18}$ Suger, dans sa Geste de Louis le Gros, exploite à son tour toutes les ressources de « cette métaphore vive » qui fait de la parole efficace de l'Eglise (anathème, prédication) l'arme véritable à laquelle toutes les autres armes sont subordonnées : «Venerabilis sancte Romane ecclesie legatus Cono, Prenestinus episcopus, innumerarum pulsatus molestia querelarum ecclesiarum, pauperum et orphanorum devexationum, ejus tirannidem muchrone beati Petri, anathemate scilicet generali detruncans, cingulum militarem ei licet absenti decingit, ab omni honore tanquam sceleratum, infamatum, christiani nominis inimicum omnium judicio deponit. Tanti itaque concilii rex exoratus deploratione, citissime in eum movet exercitum et clero, cui semper humillime herebat, comitatus, Creciacum munitissimum castrum divertit, armatorum potentissima manu, quin potius divina, inopinate castrum occupat, turrim fortissimam ac si rusticanum tugurium expugnat, sceleratos confundit, impios pie trucidat et quos, quia inmisericordes offendit, inmisericordes detruncat. Videres castrum ac si igne conflari infernali, ut fateri non differres : 'Pugnabit pro eo orbis terrarum contra insensatos' » (Sap 5, 21) (Le vénérable légat de la sainte église romaine, Conon, évêque de Palestrina, vivement ému par les innombrables plaintes des églises et les souffrances des pauvres et des orphelins, abattit sa tyrannie en le frappant de l'épée mordante de saint Pierre, c'est à savoir d'un anathème général, le
} 
le reflet dégradé d'un modèle plus haut incarné par la parole efficace des clercs, «glaive spirituel » qui s'abat ${ }^{19}$ sur les ennemis de l'Eglise au travers des armes des milites.

Tel est l'un des mécanismes d'expression de l'«auxiliarité » du laïc par rapport au clerc, topique dans le discours réformateur ${ }^{20}$. Telle est l'une des structures qui fondent la reconnaissance humiliée de l'état de laïc par rapport à l'état de clerc, selon un système d'homologies complexes étagées depuis la volonté divine qui les ordonne: c'est la potestas de la parole qui frappe, en une métaphore souvent bien plus que vive, tandis que la potentia des armes n'en est que l'ombre diminuée.

\section{LA PAROLE, UN MOTIF PARTAGE PAR LES DISCOURS}

Il peut paraître superflu, à ce point du raisonnement, de rappeler que le motif de la parole et de ses pouvoirs, ainsi que les portraits des clercs et des laïcs à la peinture desquels ce motif participe, sont pris en charge par le discours ecclésiastique en latin qui domine toute la production écrite jusqu'au début du XII ${ }^{\mathrm{e}}$ siècle : ce sont les énoncés des clercs qui, dès les premiers textes, sont «de droit, dépositaires de la parole vertueuse ${ }^{21} »$. Ce sont, comme l'a montré l'enquête d'Anita Guerreau-Jalabert, les énoncés ecclésiastiques qui prennent en charge le paradigme de la parole et de la parabole christiques et le projettent de manière complexe sur toute parole chrétienne, avant que les langues romanes ne déploient leurs propres constructions sémantiques ${ }^{22}$. Et ce sont donc certains de ces mêmes énoncés ecclésiastiques, les discours des réformateurs, qui ont placé l'efficacité de la parole au centre d'un idéal de construction sociale et d'organisation politique de la société

dépouilla, quoiqu'absent, du baudrier de guerrier, le déclara, en vertu d'un jugement unanime, déchu de tous ses honneurs comme criminel, infâme, ennemi du nom chrétien. Cédant à la prière et aux plaintes d'un si grand concile, le roi met très vite son ost en mouvement contre lui et, accompagné du clergé, auquel il fut toujours très humblement attaché, se tourne vers le château solidement fortifié de Crécy. Grâce à la main très puissante de ses hommes, que dis-je, bien plutôt grâce à celle de Dieu, il s'en empare à l'improviste, prend d'assaut la tour, qui était très forte, tout comme il eût fait d'une cabane de paysan, confond les criminels, massacre pieusement les impies ; ceux auxquels il se heurte parce qu'ils sont sans pitié, sans pitié il les abat. Si vous aviez vu le château, vous l'eussiez cru embrasé du feu de l'enfer et, tout de suite, vous eussiez reconnu la vérité de cette parole : «Tout l'univers combattra pour lui contre les insensés ») (Vie de Louis VI le Gros, édition et traduction, H. Waquet, Paris, 1929, XXIV, p. 174-177, à propos de Thomas de Marle). La notion de militia permet plus largement de composer ce système hiérarchique au moyen d'une reprise des mêmes termes (militia spiritualis et militia saecularis; vocabulaire des armes, etc....): «Pour les ecclésiastiques, appeler les chevaliers des milites, c'est se dire parallèles et supérieurs à eux : une milice cache l'autre, ou plutôt, elle la sous-entend », D. Barthélemy, La mutation de l'an mil a-t-elle eu lieu ? Servage et chevalerie dans la France des $X^{e}$ et XI $I^{e}$ siècles, Chapitre V : «Le mot miles et l'histoire de la chevalerie », Paris, 1997, p. 173-191, p. 190.

${ }^{19}$ Selon Rom. $13: 4$.

${ }^{20}$ «Le début du XII ${ }^{\mathrm{e}}$ siècle est [...] le temps où fut exprimée de la façon la plus rigide l'idée d'une 'auxiliarité' de la fonction princière, notamment au travers d'une volonté tenace des partisans de la réforme d'affirmer le rôle moteur de l'Eglise dans la prise en charge de la paix publique, et de confiner rois et princes dans une simple fonction d'exécution » (Y. Sassier, «L'Histoire du monastère de Vézelay», p. 165). Voir pour une vue d'ensemble : D. Iogna-Prat, «La place idéale du laïc à Cluny (v. 930-v. 1150) : d'une morale statutaire à une éthique absolue ? », dans Guerriers et moines (Conversion et sainteté aristocratiques dans l'Occident médiéval ; IX $X^{e}$-XII siècle), éd. M. Lauwers, Antibes, 2002, p. 291-316.

${ }^{21}$ C. Casagrande, S. Vecchio, Les péchés de la langue, Paris, 1991 (trad. frçse), p. 18. Les clercs ont bâti par ce biais un système de représentations de la parole, de ses pouvoirs et de ses valeurs, en amont de la grande systématisation du XIII ${ }^{\mathrm{e}}$ siècle décrite par S. Casagrande et S. Vecchio.

${ }^{22}$ A. Guerreau-Jalabert, «Parole/parabole; la parole dans les langues romanes : analyse d'un champ lexical et sémantique », dans La parole du prédicateur, dir. R. M. Dessí, M. Lauwers, Nice, 1997, p. 311339. 
chrétienne. Or au début du XII ${ }^{\mathrm{e}}$ siècle, il faut noter ce «problème pressant ${ }^{23}$ » que représente en contexte la naissance d'un corpus écrit non plus en latin, mais en langue vernaculaire. C'est, on le sait, une naissance en deux temps dont on peut préciser comme Michel Zink que la «seconde», une fois éloignée «l'ombre des lettres latines » et abandonnée la stricte fidélité de la production au genre hagiographique en particulier, est «plus soudaine que la première, plus surprenante » et que ses «suites allaient être plus fécondes ${ }^{24} »$.

Nous nous proposons de poser, à partir du motif de la parole efficace, la question du degré et des modes d'insertion du nouveau corpus en langue vernaculaire (que nous identifions un peu trop commodément comme ressortissant de la catégorie de la « littérature », en postulant ainsi une catégorie qui semble pour le moins problématique à imposer au système des discours médiévaux) dans le tissu des autres discours, tissu où il se trouve pris au moment de son émergence. Jean-Marie Fritz, qui pose cette même démarche en tête de son travail sur le discours du fou au Moyen Age central, rappelle après Michel Foucault que les discours d'une situation donnée «ne peuvent pas proférer tous les types d'énoncés possibles ${ }^{25} \gg$; ceci, en raison du système qu'ils constituent et dans lequel ils s'engendrent mutuellement, par dialogue, opposition, participation et transferts de motifs. Tout nouvel énoncé serait comme un «nœud dans un réseau ${ }^{26}{ }$, réseau de questions et de réponses qui forment système. Cette hypothèse peut se traduire en termes sociohistoriques : si nous ignorons tout, la plupart du temps, des conditions de production et de diffusion de ces nouveaux textes, certains indices textuels - le type d'héroïsme qui s'y déploie notamment - confirmeraient que nous assisterions là à une première appropriation, par le groupe des grands aristocrates laïcs, du support de l'écriture. Anita GuerreauJalabert et Michel Banniard suggèrent que la première manifestation d'une production textuelle en langue romane autonome (chansons de geste, lyrique d'oïl et d'oc, et romans, antiques puis arthuriens) pourrait bien être le résultat d'une demande, celle de l'aristocratie laïque, et d'un projet: légitimer ses valeurs, «se justifier dans l'ordre historique ${ }^{27}$ », et s'autodéfinir :

Ce qui se manifeste alors, c'est en quelque sorte une prise de parole, légitimée par l'écrit, de la part de la fraction laïque dominante ${ }^{28}$.

L'hypothèse selon laquelle les discours en présence font système signifie donc concrètement que se noueraient des rapports essentiels (de partage de motifs et de confrontation de valeurs) entre les énoncés ecclésiastiques (en latin ou en langue vernaculaire) et les nouveaux énoncés en langue vernaculaire, parce qu'il y va de la " prise de parole » et de l'autorité de ces derniers. Les travaux de John Baldwin ${ }^{29}$ ou de Jean-

\footnotetext{
${ }^{23}$ E. Gilson, La Théologie mystique de saint Bernard, Paris, 1931, p. 14.

${ }^{24}$ M. Zink, Littérature française du Moyen Âge, Paris, 1992, p. 41.

${ }^{25}$ Le discours du fou au Moyen Age. XII ${ }^{e}$-XIII ${ }^{e}$ siècles. Etude comparée des discours littéraire, médical, juridique et théologique de la folie, Paris, 1992, p. 5.

${ }^{26}$ M. Foucault, L'archéologie du savoir, Paris, 1969, p. 36.

${ }^{27} \mathrm{M}$. Banniard, « Genèse de la langue française (III ${ }^{\mathrm{e}}-\mathrm{X}^{\mathrm{e}}$ siècles) », dans Histoire de la France littéraire. T. I : Naissances, Renaissances (Moyen Age-XVI siècle), «Le français et la latinité : de l'émergence à l'illustration », dir. F. Lestringant, M. Zink, Paris, 2006, p. 9-35, p. 30.

28 A. Guerreau-Jalabert, «Le temps des créations (XI'-XIII siècle)», dans Le Moyen Âge. Histoire culturelle de la France - 1, dir. M. Sot, J.-P. Boudet, A. Guerreau-Jalabert, Paris, 2005, p. 144.

${ }^{29}$ Les langages de l'amour dans la France de Philippe Auguste, Paris, 1997 (trad. frçse).
} 
Marie Fritz ${ }^{30}$ ont construit sur cette hypothèse une confrontation archéologique spécifique des discours en présence (ecclésiastiques et non ecclésiastiques) autour d'un objet nodal que désignait son rapport à la production d'un pouvoir et d'un savoir autorisé : sexualité et folie. Mais qu'en est-il de cet autre problème particulièrement sensible, celui du pouvoir des mots et de l'efficacité ou de la fonction opérative de la parole, qui dessine les contours du pouvoir spirituel des ecclésiastiques ? Quels sont le degré et les modes de participation du corpus émergent, non ecclésiastique donc, à des questions qui touchent au statut des paroles sacramentelles et de la prédication, qui concernent ainsi les plus grands rituels chrétiens et le fonctionnement même du sacré et assurent de fait la supériorité éthique mais aussi sociale des « hommes spirituels »? Que font les énoncés dits « littéraires » quand ils s'emparent de cet ensemble de motifs - parole efficace, armes, amour, pouvoirs... - qui fondent tout à la fois la dépendance et l'«auxiliarité » des grands aristocrates laïcs, de leurs fonctions sociales et de leur histoire ${ }^{31}$ ? Le Thersite de Shakespeare a beau jeu de dire, comme le relève Jean-Yves Tilliette, que «la guerre et la fornication, il n'y a vraiment qu'elles qui soient toujours à la mode ${ }^{32} »$, il ne revient pas sans doute au critique contemporain d'endosser le rôle de ce personnage... Poser l'émergence des nouveaux textes comme un fait social bouleversant en contexte implique au contraire de mettre à la question leurs grands thèmes et leur inscription particulière dans le tissu des discours du Moyen Age central, sans postuler dès l'abord et exclusivement leur gratuité ou leur fonction parodique et/ou divertissante.

Il se trouve que la jeune littérature de langue romane traduit au début du XII ${ }^{\mathrm{e}}$ siècle, dans ses premières réalisations, comme « une focalisation symbolique sur les armes, par rapport aux autres instruments du pouvoir noble ${ }^{33} »$. C'est dans la guerre (contre le sarrasin ou le seigneur rebelle) que le chevalier et le roi, Charlemagne ou Louis, entrent en scène dans la plupart des chansons de geste du XII ${ }^{\mathrm{e}}$ siècle : Chanson de Roland, Couronnement de Louis, Charroi de Nîmes, Chanson de Guillaume, etc.... Ces textes semblent donc bien reprendre la proposition de la Vie d'Isarn et d'un certain discours réformateur, selon laquelle les laïcs sont avant tout des rhinocéros armés de la corne de leur potentia, et voués à la défense armée de l'Eglise... La première explication de l'insertion massive ${ }^{34}$, dans ces textes, d'un motif aussi problématique que le «portrait du grand aristocrate laïc » en miles, cette fois pour en faire un héros, et non plus un rhinocéros, paraît relever, comme l'a noté Jean Flori, du fait que l'aristocratie guerrière «entendait [...] avoir l'initiative et le contrôle < de la guerre sainte>, l'intégrer elle-même dans son propre système de valeurs sans se laisser confiner dans une position subordonnée, dans la mouvance de l'Eglise ou de la papauté ${ }^{35} »$. L'historien ajoute par ailleurs qu'aucune chanson ne décrit une croisade véritable : l'accent est mis plutôt « sur la guerre entreprise par des chevaliers pour le service de leur roi, pour

${ }^{30}$ Le discours du fou.

${ }^{31}$ Y. Sassier, «L'Histoire du monastère », p. 165.

${ }^{32}$ Citation relevée dans L'Iliade. Epopée du XII siècle sur la guerre de Troie, dir. F. Mora, Turnhout, 2003, «Introduction », p. 5-40, p. 35.

${ }^{33}$ D. Barthélemy, La Chevalerie. De la Germanie antique à la France du XII siècle, Paris, 2007, p. 253.

${ }^{34}$ Hormis bien sûr l'explication par le recours à la topique nécessaire à la construction du genre, ici épique, que nous laissons de côté dans notre analyse : cfr F. Goyet, Penser sans concepts : fonction de l'épopée guerrière, Paris, 2006.

${ }^{35}$ J. Flori : «Le Pape, l'Ermite et le chevalier. Les métamorphoses d'un thème de croisade : l'assistance aux chrétiens opprimés, des chroniques aux chansons de geste », dans Croisade et chevalerie. $\mathrm{XI}^{e}-\mathrm{XII}^{e}$ siècles, Paris-Bruxelles, 1998, p. 243-258, p. 258. L'auteur ajoute : «Cette métamorphose traduirait-elle la volonté plus ou moins inconsciente de minimiser le rôle initiateur de la papauté et du clergé, même dans sa forme très populaire de l'érémitisme, et de valoriser au contraire l'initiative laïque, celle des chevaliers ; non seulement dans la réalisation des opérations de secours aux chrétiens opprimés, mais également dans son organisation, voire dans sa décision? » (p. 252) 
la conquête de terres sur l'ennemi infidèle sans intervention de la papauté, sans abandon, même partiel, du cingulum militiae ${ }^{36}$ ». La critique a depuis longtemps noté, de même, que la chanson exclut en grande partie de son univers les personnages de clercs et de fait, nombre d'occasions de mettre en scène leur parole efficace, notamment sacramentelle ${ }^{37}$, mais aussi toute parole de prédication propre à incarner une militia spiritualis... Les rares interventions des «hommes spirituels » dans cet univers sont souvent même marquées au fer rouge de la déviance la plus charnelle ${ }^{38}$ quand leurs interventions rituelles ne sont pas pour le moins détournées par rapport aux prescriptions des ordines ${ }^{39}$.

Ce faisceau d'indices incite à penser que nous sommes là en présence d'un système cohérent de motifs (fonction guerrière et parole) : nous aurions affaire, plus qu'à des «thèmes épiques » universels ou strictement transitifs par rapport à l'événement de la croisade, à de véritables questions, prises en charge par les énoncés nouveaux comme elles l'étaient déjà par les énoncés ecclésiastiques. C'est à travers ces questions que les énoncés nouveaux prendraient place au sein des discours. La parole efficace, liée comme elle l'est à la représentation de l'infériorité et de l'auxiliarité de la fonction guerrière, elle-même donnant ses contours au statut du grand laïc, serait une de ces questions, partagées par tous les énoncés et discours : cela laisse augurer que la récupération, par la chanson de geste, d'un personnage de miles, du motif des armes et du motif de la parole, engage une problématique bien plus large que celle de la seule guerre contemporaine, intérieure ou extérieure.

C'est à partir de cette hypothèse que nous procèderons à un essai de repérage de la parole efficace et de son rapport avec le motif des armes dans les chansons, de manière à amorcer une évaluation des contours donnés en ces textes au portrait du grand aristocrate laïc et à tenter de le confronter avec le rhinocéros-miles du discours réformateur. Qu'en est-il par exemple du serment et du gab des chansons? Les travaux fondateurs de Christiane Marchello-Nizia sur les formes du serment épique ${ }^{40}$ ont montré déjà qu'il y avait là un gisement fondamental de paroles opératoires, qui font, au sens propre, l'action $\mathrm{du}$ héros guerrier. Nous reviendrons à ce premier gisement, mais voudrions travailler d'abord sur la deuxième forme de parole que nous avons évoquée : le gab, parole que son caractère ludique rend problématique en raison du pouvoir opératoire aléatoire qui l'accompagne et de son élaboration linguistique complexe. Nous allons tenter de décrire le plus fidèlement possible cette forme de parole, sans chercher à la définir a priori ${ }^{41}$. Une

\footnotetext{
${ }^{36}$ Ibidem, p. 254-255.

${ }^{37}$ Comme le montrent les différentes mises en scène de la mort du héros guerrier, construites sans recourir aux rituels cléricalisés.

${ }^{38}$ Dans le Couronnement de Louis, éd. E. Langlois, Paris, 1925, Guillaume punit « le clergié qui a lor seignor falsent » (laisse XLII, v.1763) et qui « por aveir ont le mal plait basti » (laisse XL, v.1694). Le pape qui demande l'aide du chevalier lui promet en récompense non seulement le paradis mais aussi, « en trestot ton eage », de pouvoir «mangier char » et «feme prendre tant come il t'iert corages » (laisse XVIII, v.390391)...

${ }^{39}$ On peut par exemple admirer la façon dont le couronnement de Louis se déroule sous l'autorité active non de l'archevêque pourtant présent, mais de l'empereur Charlemagne (Couronnement de Louis, laisses III et suivantes). Cfr sur ce point D. Boutet, Charlemagne et Arthur ou le roi imaginaire, Paris, 1992, en particulier p. $56 \mathrm{sq}$.

${ }^{40}$ Dire le vrai : l'adverbe « si » en français médiéval. Essai de linguistique historique, Genève, 1985.

${ }^{41}$ Nous ne rappellerons ainsi l'histoire générale du mot et de la pratique, telle que J. L. Grigsby l'analyse dans «Le gab dans le roman arthurien français », Actes du XVI Congrès International arthurien, tome 1 (1985), p. 257-272 et dans The "Gab» as a Latent Genre in Medieval French Literature : Drinking and Boasting in the Middle Ages (Medieval Academy Books, 153), Cambridge, 2000, qu'une fois notre description achevée.
} 
chanson, transmise par un seul manuscrit aujourd'hui disparu, et à la datation discutée ${ }^{42}$, se détache par sa manière insistante et particulière de mettre en scène le gab: il s'agit du Voyage ou Pèlerinage de Charlemagne à Jérusalem et à Constantinople ${ }^{43}$, qui reprend le motif du voyage de l'empereur dans les deux cités lointaines de Jérusalem et de Constantinople $^{44}$. C'est sur cette chanson, largement étudiée déjà, que nous appuierons d'abord notre tentative de description des formes et de l'efficacité de la parole gabeuse.

\footnotetext{
${ }^{42}$ Un historien spécialiste de l'Orient latin a apporté quelques preuves à partir de la description du « marché de Jérusalem» (v.209-212) décrit dans la chanson : J. Richard, «Sur un passage du Pèlerinage de Charlemagne : le marché de Jérusalem », Revue belge de philologie et d'histoire, 43-2 (1965), p. 552-555. Il conclut à une datation dans le second quart du XII ${ }^{\mathrm{e}}$ siècle, comme P. Aebischer, Les versions norroises du "Voyage de Charlemagne à Jérusalem ». Leurs sources, Paris, 1956. Cfr les propositions de C. Rossi dans sa thèse, Ja ne m'en turnerai trescque l'avrai trovez. Ricerche attorno al ms. Royal 16 E. VIII, testimone unico del "Voyage de Charlemagne à Jérusalem et à Constantinople », e contributi per una nuova edizione del poema, doctorat, Université de Fribourg, Fribourg, 2005 : l'enquête, érudite et précise, sur les liens qu'entretient la chanson avec des textes produits par Saint-Denis ou recueillis à l'abbaye, des reliques qui y sont gardées, ou même avec la géographie du lieu, permet d'approfondir ces questions.

${ }^{43}$ L'édition que nous utiliserons est celle de $\mathrm{P}$. Aebischer, Le Voyage de Charlemagne à Jérusalem et à Constantinople, Genève, $1965(T L F)$. Le titre donné par le manuscrit unique (le manuscrit de la King's Library 16. E. VIII du Musée britannique) aujourd'hui disparu est le suivant: «Ci comence le livere cumment charels de fraunce voiet in ierhusalem Et pur parolz sa feme a constantinople pur vere roy hugon » (transcription par E. Koschwitz, dernier éditeur à avoir travaillé sur le manuscrit avant sa disparition en 1879 - on peut trouver cette édition avec une traduction d'A. J. Cooper dans Le Pèlerinage de Charlemagne, publié avec un glossaire, Paris, 1925 - et résolution par P. Aebischer dans son édition, p. 15). Mais cfr aussi C. Rossi, Ja ne m'en turnerai trescque l'avrai trovez et l'édition nouvelle du texte qu'elle a fournie à partir de sa thèse. Pour les débats sur le titre, voir note suivante. Pour la bibliographie, abondante, nous renvoyons à l'article que le Dictionnaire des lettres françaises. Le Moyen Age, éd. revue et corrigée par G. Hasenohr et M. Zink, Paris, 1992, consacre à ce texte. Signalons cependant : J. Horrent, Le Pèlerinage de Charlemagne. Essai d'explication littéraire, avec des notes de critiques textuelles, Paris, 1961 et pour une réponse, P. Aebischer, «Sur quelques passages du Voyage de Charlemagne à Jérusalem et à Constantinople. A propos d'un livre récent », Revue belge de philologie et d'histoire, 40-3 (1962), p. 815-843; M. Tyssens, Le Voyage de Charlemagne à Jérusalem et à Constantinople, Gand, 1978 (traduction critiques et notes), et les analyses et synthèses récentes de M. Bonafin, La Tradizione del "Voyage de Charlemagne » $e$ il "gabbo », Alessandria, 1990 ; A. E. Cobby, Ambivalent Conventions. Formula and Parody in Old French, Amsterdam and Atlanta, 1995 (chapters 4 and $5:$ p. 82 sq.) et J. L. Grigsby, The « Gab» as a Latent Genre in Medieval French Literature.

${ }^{44} \mathrm{P}$. Aebischer, Les versions norroises; J. Horrent, «Sur les sources épiques du Pèlerinage de Charlemagne », Revue belge de philologie et d'histoire, 38-3 (1960), p. 750-764. Les deux moments du voyage ont posé problème, surtout par leur disproportion (141 vers seulement pour Jérusalem) et leur différence de registre : J. Horrent maintient la thèse d'une forte unité de la chanson (voir en particulier « La chanson du Pèlerinage de Charlemagne. Problèmes de composition », dans La technique littéraire des chansons de geste. Actes du colloque de Liège, Paris, 1959, p. 413-428), alors que d'autres critiques tendent à $\mathrm{y}$ voir la réunion fortuite et maladroite de deux textes séparés. L’interprétation de J. Grisward ( Paris, Jérusalem, Constantinople dans le Pèlerinage de Jérusalem : trois villes, trois fonctions », dans Jérusalem, Rome, Constantinople : l'image et le mythe de la ville au Moyen Age, éd. D. Poirion, Paris, 1986, p. 75-82) expose les nécessités fonctionnelles de chaque lieu urbain. Mais la théorie d'une forte unité de la chanson ne résout pas la question de son titre, pas plus que celle de son sens et de ses priorités : pour J. Horrent, qui a défendu l'appellation de Pèlerinage, Charlemagne «a deux raisons de pérégriner en Orient: satisfaire sa dévotion et vérifier les dires de la reine », et le critique liégeois ajoute que « la priorité matérielle attribuée à l'épisode de Jérusalem, la prééminence accordée au sentiment religieux, nous assurent que pour Charlemagne, le voyage en Orient est avant tout un pèlerinage sur les lieux saints » (Le Pèlerinage de Charlemagne. Essai d'explication, p. 23 et p. 24). Pour P. Aebischer, au contraire, la raison du voyage est l'amour-propre blessé du roi... («Sur quelques passages », p. 820) et «il faut donc un sérieux parti-pris pour voir dans cette équipée [...] une manifestation de foi. Si bien que donner à ce récit le nom de 'pèlerinage' est une aberration, et que seule lui convient la dénomination de 'voyage' " (Le Voyage de Charlemagne, «Introduction », p. 14). Cfr aussi l'article de C. Rossi, «Le Voyage de Charlemagne: le parcours vers Jérusalem et les reliques », Critica del testo, 2:2 (1999), p. 619-653, qui reprend ce questionnement en exposant à nouveaux frais la géographie de l'itinéraire et aussi des reliques.
} 

ANALYSE

Charlemagne et ses grands partent vers Jérusalem et Constantinople en pèlerins, munis de bourdons de frênes. Ils n'ont ni «escuz ne lances ne espees trenchaunz » («ni écus, ni lances, ni épées tranchantes », v.79). Le départ de la troupe est provoqué par la reine. Charlemagne au début de cette histoire se trouve à Saint-Denis. Entouré de sa cour, il y ceint « à nouveau » sa couronne et son épée : il entraîne par la main la reine sous un olivier et lui déclare à haute voix : "Dame, veïstes onques hume dedesuz ceil/ Tant ben seïst espee, ne la corune el chef? » («Dame, avez-vous déjà vu quelqu'un/ porter aussi bien l'épée, et la couronne sur la tête ? », v.9-10). La reine alors lui répond «folement » (v.12) qu'il s'estime trop, et qu'elle sait un roi qui porte sa couronne plus bellement encore : il s'agit d'Hugon, le roi à la charrue d'or qui règne sur Constantinople. La reine tente d'expliquer sa parole : elle a seulement voulu «jouer» (v.33). Rendu fou de rage par cet échange que tous les Français ont entendu (v.18), Charlemagne réunit ses douze pairs (Olivier et Roland, Guillaume d'Orange et l'archevêque Turpin, Bérenger et Naimes, Bertrand et Bernard...) et les informe du départ : il ira d'abord à Jérusalem pour y adorer Croix et saint Sépulcre parce qu'un songe triple le lui a imposé ; puis il se lancera dans la quête de ce roi Hugon. Il ne reviendra pas avant de l'avoir trouvé.

Le récit se déroule à partir de là en deux temps ${ }^{45}$ : à Jérusalem, Charlemagne et ses douze pairs entrent en une église où Dieu aurait célébré la messe pour ses apôtres. Il s'y trouve les douze chaires des apôtres plus une, magnifique et fermée, celle où se serait assis le Christ. Charlemagne et les siens y prennent place. Le roi a le visage si «fier» (v.131) qu'un juif croit voir le Christ et les apôtres et demande à être baptisé sur le champ. Prévenu, le patriarche de la ville baptise l'empereur, en raison de cette aventure (nul n'avait jamais pris place dans la chaire fermée), du nom de «Charlemaine sur tuz reis curunez » (v.158) et lui confie treize reliques (reliques de saints martyrs, reliques de la Passion du Christ, reliques mariales) ${ }^{46}$, ce qui produit d'emblée, sur place, un miracle de guérison. Charlemagne et ses pairs résident à Jérusalem le temps d'entreprendre la construction d'une basilique et d'achever la confection d'une châsse d'or pour les reliques. Puis, ils décident de repartir ${ }^{47}$.

Charlemagne se souvient alors de son autre quête : celle du roi Hugon. Les Français passent par Jéricho, où ils prennent des palmes. Devant les reliques qui accompagnent le convoi, les miracles se multiplient : des malades sont guéris, et les eaux des cours d'eau s'écartent. La troupe arrive à Constantinople : c'est une « citez vaillant » (v.262) brillant de mille feux, cernée de vergers incroyables. Entre autres merveilles, les chevaliers présents, magnifiquement vêtus et au nombre de vingt mille, se divertissent en compagnie de treize mille jeunes filles. Le roi Hugon, équipé somptueusement, laboure avec sa charrue d'or. Il accueille Charles et ses pairs et les conduit au palais : les somptuosités du palais et ses mécanismes merveilleux (des statues animées) suggèrent à Charlemagne que, par comparaison, sa propre «manantise » («possessions », v.363) ne vaut guère. Et voilà que

${ }^{45}$ Voir note 44.

${ }^{46}$ Cfr les travaux de C. Rossi : sa thèse et aussi «Le Voyage de Charlemagne : le parcours vers Jérusalem », qui analyse particulièrement la localisation des reliques au $\mathrm{XII}^{\mathrm{e}}$ siècle, et donne une interprétation tout à fait intéressante des miracles accomplis.

${ }^{47}$ Le «patriarche » les supplie alors de bien vouloir défendre la terre sainte contre les Sarrasins et les païens (v.224-225) qui veulent détruire la chrétienté. Charlemagne lui en fait la promesse : «Sa fei si l'en plevit» (v.228). Le texte évoque alors la guerre en Espagne et la mort de Roland et souligne que Charlemagne accomplira sa promesse («ben en guardat sa fei », v.231). 
les pairs sont terrorisés par les merveilles du palais, qui se met à tourner sur lui-même tandis qu'un orage violent se fait entendre à l'extérieur. Les Francs dînent somptueusement (venaison, épices, vin et musique) tandis qu'Olivier s'enflamme, à table, pour la fille d'Hugon. Quand ils se retirent, seuls, avec du vin, dans leur chambre somptueuse, les Français commencent à «gabber» (v.446). A vrai dire, deux d'entre eux avaient déjà commencé, mais silencieusement : Guillaume d'Orange, à propos de la charrue d'Hugon, et Olivier, à propos de la fille du roi... Dans la chambre, en revanche, chacun parle haut et clair. Un espion, placé là par le roi méfiant, les écoute et n'en peut mais, réagissant en aparté à chacun des gabs proférés.

Que sont exactement ces gabs ? Tout d'abord, la parole gabeuse est collective, avant que Charlemagne ne la prenne en charge :

Et dist li uns a l'altre : «Veez cum grant beltet !

Veez, cum gent palais et cum forz richetet !

Pleüst al rei de glorie de sainte majestet

Carlemaine mi sire les oüst recatet

U conquis par ses armes en bataile champel !»

Et lur dist Carlemaine : «Ben dei avant gabber» (v. 448-452)

(Et l'un dit à l'autre : « Voyez cette grande beauté !

Voyez ce si noble palais, et cette si évidente puissance !

Si seulement il plaisait au Roi de gloire, de sainte majesté,

Que Charlemagne, mon seigneur, ait acheté tout cela

Ou l'ait conquis par les armes au cours d'une bataille rangée !»

Et Charlemagne de leur dire : «Je dois gabber, maintenant !»)

L'empereur déclare qu'il coupera en deux jusqu'à la selle le meilleur chevalier d'Hugon, et d'un seul coup d'épée, quand bien même il aurait revêtu deux casques et deux hauberts ; Roland soufflera dans un olifant une tempête telle qu'elle détruira toutes les portes de la ville et dévêtira Hugon en lui arrachant les poils de sa barbe ; Olivier fera l'amour cent fois dans la même nuit à la fille du roi Hugon : dans le cas contraire, il jure «par covent » («par promesse ») de se laisser couper la tête ; Turpin, l'archevêque, courra aussi vite que trois destriers de Hugon lâchés au galop, avant d'en enfourcher un et de jongler avec quatre pommes sans en laisser choir une seule : que Charlemagne lui crève les yeux s'il en laisse tomber une ; Guillaume d'Orange propose de soulever l'énorme escarboucle qui éclaire la chambre, et de la jeter sur le palais pour en briser un $\operatorname{mur}^{48} \ldots$ Au matin, informé par

\footnotetext{
${ }^{48}$ Ogier fera de même avec le pilier qui soutient tout le palais, et en jettera le palais à terre ; le vieux Naimes brisera de ses seuls muscles le fort haubert dont on l'aura revêtu ; Béranger se jettera d'une tour sans être blessé sur des épées plantées pointes en l'air ; Bernard fera déborder la rivière, qui noiera le royaume et poussera Hugon à fuir dans sa tour ; Ernald se plongera dans une cuve de bois et de plomb fondu, et attendra que le plomb prenne : il s'en libèrera ensuite ; Aimery mangera dans l'assiette du roi Hugon son poisson et boira son vin, avant de le frapper par derrière, à sa table ; Bertrand frappera si fort deux écus l'un contre l'autre qu'il fera fuir tout être vivant, homme et bête, de la forêt du roi ; Gevin lancera de loin un lourd épieu,
} 
l'espion et en colère, Hugon attend de pied ferme les Français, sommés de réaliser effectivement leurs gabs: Charlemagne tente de rappeler, mais en vain, que telle est la coutume des Francs qu'avant d'aller se coucher, et après boire, ils « se giuent et gabent » (« ils se divertissent et gabent», v.655).

L'empereur réunit ses pairs à part, et tous se mettent à genoux devant la châsse des reliques pour prier Dieu : un ange apparaît, qui garantit à Charlemagne que Dieu l'aidera à accomplir les gabs, tout en lui donnant l'ordre de ne plus jamais «gaber » quiconque («ne gabez ja mès hume, ço cumandet Christus ! »: « ne gabez plus personne, c'est ce que vous ordonne le Christ! », v.676). Plein de bravoure à nouveau, Charlemagne repart auprès d'Hugon, afin de lui lancer son défi promissoire, à propos des gabs énoncés : «Nus les aamplirun »( «nous les accomplirons », v.690). Olivier commence : Hugon lui offre sa fille pour qu'il accomplisse son $g a b$. Or, si le chevalier ne fait l'amour que trente fois dans la nuit à la fille du roi, celle-ci, conquise, jure à son père qu'il l'a bien fait cent fois. Guillaume puis Bernard s'exécutent à leur tour : un mur du château est détruit, l'eau inonde la ville. Hugon demande grâce à Charlemagne, se soumet à lui et l'eau, à la prière de Charlemagne, se retire. Le roi Hugon renonce à demander la réalisation des gabs :

«A feiz, dreiz emperere, jo sai ke Deus vus aime.

Tis hom voil devenir : de tei tendrai mun regne,

Mun tresor te durrai, si l'amenrai en France. » (v.796-799)

(«Légitime empereur, je sais pour finir que Dieu vous aime.

Je veux devenir ton homme : je tiendrai mon royaume de toi ;

Je te donnerai mon trésor : je l'amènerai en France. »)

Charlemagne accepte alors la soumission d'Hugon (mais non son trésor) :

«Ore estes vus mis heoms, veant trestuz les voz.» («Vous êtes maintenant mon homme, tous les vôtres en sont témoins. », v.803)

Puis il propose une assemblée solennelle, au cours de laquelle les deux rois porteront couronne. Or, le roi Hugon porte la sienne «plus basement un poi :/ Karlemaine fud graindre de plein ped e .iii. pouz» («un peu plus bassement: Charlemagne était plus grand, d'un plein pied et de trois pouces », v.810-811). La reine en vantant ce roi, disent alors les Français, «folie dist e tord » («a dit une folie et une chose injuste », v. 813). Tout le monde rentre en France et le texte souligne en conclusion deux faits : premièrement, la domination incontestable de Charlemagne - et obtenue sans bataille - sur tout l'espace oriental de la chrétienté : "Mult fu lied e joius Carlemaine li ber,/ Ki tel rei ad cunquis sanz bataille campel» («Charlemagne le vaillant fut extrêmement heureux et joyeux d'avoir conquis un tel roi sans bataille rangée », v.858-859); deuxièmement, l'arrivée en France des reliques et leur répartition dans le royaume (les clous et la couronne reposeront

qui atteindra un denier posé sur un autre denier sans faire bouger ce dernier cependant. Puis, il courra de telle sorte que l'épieu n'aura pas le temps de toucher la terre.... 
à Saint-Denis $)^{49}$. Enfin, Charlemagne pardonne à la reine «pur l'amur del sepulcre que il ad aüret» («pour l'amour du Sépulcre qu'il a adoré », v.870), selon le dernier vers de la chanson.

\section{ANALYSE LINGUISTIQUE DU GAB}

Le gab est la forme de la parole qui semble a priori la moins efficace, la plus ludique et la moins propice à illustrer la question du pouvoir des mots. C'est à son analyse linguistique que nous allons nous consacrer à présent, au moins pour le cas particulier de la chanson qui nous occupe, avant de rappeler quelles sont les autres manifestations de cette parole.

Le gab dans le Voyage est d'abord une phrase déclarative à la première personne ancrée dans une situation de colère et d'humiliation. Les passages du texte consacrés aux gabs s'ouvrent, rappelons-le, sur un souhait non réalisé au subjonctif imparfait: les preux regrettent que Dieu n'ait point encore voulu que Charlemagne s'empare par les armes ou par un autre type de conquête des richesses et du royaume d'Hugon. La «cérémonie » des gabs est alors, comme en réponse, ouverte par Charlemagne lui-même (v.448-452). Le gab est dès lors un jeu de langage fortement virtualisé. En premier lieu, il abstrait de la situation de communication le destinataire qu'il vise : les Français attendent le secret de leur chambre pour gaber, et les gabs publics d'Olivier et de Guillaume ont été énoncés à voix basse, précise le texte. Le jeu obéit à un rituel mécanique, laisse après laisse : ici, par exemple, le premier personnage, Charlemagne, déclare solennellement qu'il ouvre l'échange des gabs («Ben dei avant gabber», v.453) puis demande à un autre personnage de gaber à son tour («Gabbez, bel neis Rolland!» : «Gabez Roland, mon cher neveu ! », v.469), ce que ce personnage octroie («Volenters, dist il, sire : tut al vostre comand !»: «Volontiers, sire, dit-il, je suis entièrement à vos ordres !", v.470). Les autres gabs obéissent au même cérémonial, rythmé par le style formulaire propre à l'écriture épique ${ }^{50}$. A ce rituel qui fonde le jeu, s'ajoutent l'ivresse accompagnant l'énoncé des gabs et plus largement les circonstances collectives et festives (le groupe des «amis » réunis après un festin et jaloux de la richesse matérielle d'Hugon). Au caractère ludique se superpose un exercice collectif d'auto-définition, par chaque pair, de ses «qualités » propres, mais la vantardise $^{51}$ énoncée donne aussi bien les contours identitaires de la communauté des «Francs » par opposition aux «autres ». Le contenu du gab est de plus extraordinaire et intenable, ce qui le virtualise encore, puisque chaque gab consiste pour celui qui le profère à garantir, voire à promettre, qu'il va accomplir un acte normalement irréalisable à l'encontre d'Hugon.

Mais chaque gab est par ailleurs décliné au futur de l'indicatif, tandis que le subjonctif présent à valeur injonctive est utilisé pour énumérer les conditions de réalisation du gab ( $g a b$ de Roland : «Dites al rei Hugun me prest sun olivant,/ Pus si m'en irrai jo la defors en cel plain... », soit «Dites au roi Hugon de me prêter son olifant, puis je me rendrai là, dehors, en cette plaine...», v.471-472). Or le subjonctif présent exprime un potentiel du

\footnotetext{
${ }^{49}$ Mais cfr les analyses de C. Rossi pour une analyse exacte de ces reliques « épiques ».

${ }^{50}$ Voir en particulier : M. Bonafin, La Tradizione del «Voyage de Charlemagne »e il « gabbo » et A. E. Cobby, Ambivalent Conventions, qui analyse avec précision le rapport du «style formulaire » épique avec le rituel du $g a b$, et aussi ses liens avec la tradition « romanesque ». Pour J. L. Grigsby, le Voyage offrirait une sorte d'exemple unique de gab comme genre littéraire.

${ }^{51}$ D'où l'un des débats sur le sens du mot gab, entre J. L. Grigsby, The « Gab » as a Latent Genre, qui voit dans la «vantardise » l'essence de cette parole, tandis que G. S. Burgess (qui a édité et traduit le texte : Le Pèlerinage de Charlemagne, Edinburgh, 1998) y lit plutôt une «farce », une «plaisanterie », insistant donc sur le côté ludique. Nous y reviendrons.
} 
présent, établissant une probabilité comme l'énoncé au futur, puisque l'«emploi basique du futur en fait un temps prospectif indiquant un procès à réaliser, ancré dans le virtuel ${ }^{52}$ », mêlant donc valeurs temporelles et valeurs modales ${ }^{53}$. Plus précisément, cependant :

Un procès projeté dans l'avenir est envisagé avec une certaine part d'hypothèse et d'incertitude. Avec le futur simple, la charge d'hypothèse est minimale, et, même si la réalisation du procès n'est pas avérée, sa probabilité est très grande ${ }^{54}$.

La situation, de ce point de vue, est identique en français moderne et en ancien français, comme le rappelle Claude Buridant qui oppose le futur de l'indicatif au conditionnel : "Quand la charge d'hypothèse dépasse le probable, on a affaire au futur catégorique $^{55} \gg$. A partir de là, l'énoncé au futur (ou au subjonctif présent) peut servir à réaliser différents types d'actes de langage directs, selon les catégories transmises par la philosophie analytique anglaise, de John L. Austin à John R. Searle ${ }^{56}$ :

Dans plusieurs cas, le futur permet au locuteur d'accomplir une action tournée vers l'avenir, qui implique généralement le destinataire. Le futur sert à accomplir trois types d'actes de langage : injonction, promesse, prédiction ${ }^{57}$.

De fait le gab, parce qu'il est aussi un énoncé déclaratif au futur de l'indicatif ou au subjonctif injonctif, peut entrer dans ces catégories d'actes de langage dont seuls «la situation et le contexte » peuvent spécifier le sens (de l'injonction à la promesse, de l'atténuation à la prédiction... $)^{58}$ et qui ont pour caractéristique principale de modifier le lien avec l'allocutaire par leur force illocutoire: de ce point de vue, il relèverait d'une parole vouée à s'aamplir, s' «accomplir », comme le prouve ce relevé qui place le gab au contact du verbe (en position de sujet) dans les paroles du roi Hugon :

Si ne sunt aampli li gab si cum il distrent... (Si ne sont pas accomplis les gabs, tout comme ils les ont prononcés..., v.632)

S'or ne sunt aampli li gab que vus deïstes... (Si maintenant ne sont pas accomplis les gabs que vous avez prononcés..., v.646) ${ }^{59}$

\footnotetext{
${ }_{53}^{52}$ C. Buridant, Grammaire nouvelle de l'ancien français, Paris, 2000, p. 363.

${ }^{53}$ «Le futur simple peut se charger de différentes valeurs modales associées à l'avenir », Grammaire méthodique du français, M. Riegel, J.-C. Pellat, R. Rioul, Paris, 1994, p. 313.

${ }_{55}^{54}$ Ibidem, p. 312.

${ }^{55}$ Grammaire nouvelle de l'ancien français, p. 263. L'auteur signale que le mode de formation périphrastique- des formes synthétiques du futur de l'indicatif et du conditionnel dans une large partie de la Romania (et même dans un ensemble assez important de langues selon Claude Hagège) témoignerait de ces

${ }^{56}$ J. L. Austin, Quand dire, c'est faire, Paris, 1970 (trad. frçse) ; J. R. Searle, Les actes de langage, Paris, 1972 ; O. Ducrot, Dire et ne pas dire, Paris, 1972 ; F. Récanati, Les énoncés performatifs, Paris, 1981.

${ }^{57}$ Grammaire méthodique, p. 313.

${ }^{58}$ Ibidem, p. 313.

${ }^{59}$ On peut aussi citer la phrase d'Hugon affolé qui renonce, à la fin du texte, à demander la réalisation des autres gabs : «Si tuz sunt aampli, ja n'ert jur ne me plaigne !» («Si tous sont accomplis, je serai bon pour me plaindre toujours! », v.801).
} valeurs. 
Puis dans la promesse de Charlemagne qui lui répond :

Nus les aamplirun, ne puet remaner mie (Nous les accomplirons, sans discussion possible, v.690)

Le terme gab complète de même les verbes asaier (v.516), cumencer (v.529), demustrer (v.552, 578), dans la parole de l'espion qui craint, ainsi, leur réalisation effective, mais aussi dans la parole de l'ange envoyé par Dieu («Va, si fas cumencer, ja ne t'en faldrat uns ! », « Va, fais-les commencer : il ne t'en manquera aucun ! », v.677).

Le gab, au départ abstrait de la situation d'énonciation par un système précis de virtualisation (le secret, le jeu, le caractère irréalisable...), est appelé de fait à s'actualiser et ce, d'autant plus aisément que sa structuration linguistique, comme on vient de le voir, permet cette actualisation. D'abord, l'allocutaire intervient dans le jeu qui ne supposait justement pas sa présence : par le biais de l'espion, puis directement. Et le Pèlerinage met alors en scène plusieurs niveaux possibles de réaction de l'allocutaire face à ce qui devient peu à peu acte de langage. Dès lors, il convient d'interroger le succès ou l'échec de l'acte langagier engagé, et de prendre la mesure de sa performativité. Pour réussir, un acte de langage suppose, comme le définit John L. Austin, l'acquiescement de l'allocutaire (et sa reconnaissance de l'intention du locuteur), mais aussi le «scénario » (mis au point par le locuteur et par le moyen des conventions de la langue) à partir duquel une répartition complexe des rôles, des droits et des devoirs respectifs dans la situation d'énonciation s'établit. L'acte de langage repose ainsi, au moment de l'énonciation, sur une convention implicite qui associe fermement telle forme donnée à la parole (mode impératif, futur de l'indicatif...) à tel acte de langage. Mais même si la situation est contrôlée par le locuteur, ce scénario peut parfaitement lui échapper au moment de la réception.

Or dans le cas du gab mis en scène par notre chanson, les locuteurs sont dépossédés de tout contrôle sur le scénario énonciatif, puisque le gab est proféré par eux à la fois sur le mode du jeu et sur le mode de l'insulte, de la menace jusqu'au serment et enfin, de la vantardise : ils font comme si le destinataire était présent, d'une part, et construisent la valeur collective et identitaire de leur groupe, d'autre part, autour d'actes qu'ils s'engagent à accomplir en allant parfois jusqu'à asserter cette réalisation. Cette indécision s'ajoute à l'extrême instabilité de l'effet de l'acte, par nature imprévisible, comme le montre la mise en scène de l'espion. De fait, droits et devoirs normalement établis par l'acte de langage, au moins à son niveau illocutionnaire, restent flous et incontrôlables pour tous les acteurs.

Définissant clairement cette ambiguïté constitutive dès l'énonciation même du gab, Charlemagne tente de suggérer ${ }^{60}$ au roi Hugon que les gabs entrent dans la catégorie des énoncés non pourvus d'une force illocutoire et qu'ils ne sauraient participer à la construction d'un «univers de croyance ${ }^{61} »$ : ils relèveraient en effet de la catégorie sémantique «folage»versus «saver» («folie»versus «sagesse», v.656) et en cela, n'ont pas à être impliqués dans une situation d'énonciation intégrant des destinataires autres que le groupe des locuteurs. Autrement dit, ils appartiendraient aux seuls locuteurs et à un jeu privé. Le contredisant radicalement, Hugon refuse au contraire que ces énoncés soient détachés de la situation d'énonciation qui l'inclut en tant qu'objet. Il fait basculer (pour le défi et la vengeance) ces paroles dans l'établissement possible, potentiel, d'un

\footnotetext{
${ }^{60}$ Comme avait tenté de le faire la reine au début du texte.

${ }^{61}$ Pour ces glissements sémantiques et logiques, voir R. Martin, Langage et croyance. Les « univers de croyance » dans la théorie sémantique, Bruxelles, 1987.
} 
« univers de croyance » dont les Français en gabant auraient affirmé la vérité ou dont ils auraient tenté d'imposer la vérité, ce par quoi, précisément, il se sent insulté. En cela, l'acte de langage s'accomplit pleinement. Hugon réengage les gabs dans la situation d'énonciation dans laquelle il est réintégré : les gabs sont un acte de langage agissant sur lui et donc, une insulte. Puis, dans un deuxième temps, pour sa vengeance, Hugon lance un défi en demandant la réalisation des gabs (aamplir est le verbe qu'il répète) : il fait glisser pour ce faire les gabs dans la catégorie «mençunge » versus « veir » (v.733), comme son espion. En la matière, sa réaction est moins transparente que celle de l'espion, sur qui l'acte de langage fonctionne directement comme promesse $e^{62}$ et prédiction, voire serment asserté : lui n'en perçoit pas, comme Hugon d'ailleurs, la dimension ludique mais craint (contrairement à Hugon sur lequel le texte ne nous renseigne pas) de voir s'accomplir les gabs parce qu'il y lit un acte de langage pourvu d'une charge de probabilité maximale. La réussite pleine du gab sur ce premier auditeur s'explique aussi par le fait que le gab peut être accompagné d'un «serment épique»: plusieurs des personnages s'engagent comme asserteurs légitimes de leurs gabs sur leur vie même ou leur intégrité physique ${ }^{63}$, comme l'archevêque Turpin qui offre ses yeux à l'empereur en cas d'échec dans sa jonglerie... Le gab, jeu privé, vantardise identitaire, insulte inutile, devient insulte efficace, assertion, et même serment sans solution de continuité ${ }^{64}$.

Mais ce qui nous intéresse avant tout, c'est que cette hypothèse de l'espion (utilisée par Hugon) selon laquelle le gab est une parole intégrée dans le système de la véridiction, comme toute parole efficace, est confirmée par la fin de notre texte : les gabs se réalisent. Dieu répond immédiatement et se compromet dans leur accomplissement, une fois que les Francs en ont appelé à lui devant les reliques obtenues à Jérusalem ${ }^{65} . .$. Nous y reviendrons. Reste à cette étape de notre analyse qu'à la reine autant qu'à l'empereur et aux douze preux, mais aussi au destinataire, le gab chargé de sa force assertive et opératoire a échappé sans retour possible. Nul n'a interprété correctement ou plutôt complètement le gab.

Le gab, parole n'appartenant pas au système de la véridiction par son contenu a priori intenable et par la mise à distance qui préside à son énonciation (le jeu), s'y trouve donc ramené parce que la situation d'énonciation l'actualise en menace, promesse et/ou en assertion et que quelque chose, au-delà de ceux qui le profèrent ou l'entendent, lui confère un pouvoir immédiat, pouvoir que ni les locuteurs, ni l'allocutaire ne sont ici en mesure de contrôler ou prévoir. La capacité, propre semble-t-il au gab, d'appartenir à la fois à la catégorie du jeu, à celle de l'insulte et à celle de l'assertion, voire à celle du serment qui lie l'énonciateur à la réalisation de l'acte, interdit d'autant plus toute simplification de sa définition que s'y joue ainsi ce pouvoir réellement incontrôlable et que s'y enracine une causalité qui ne concerne jamais que partiellement les locuteurs. Tout cela explique que la traduction du terme soit très périlleuse et très discutée, comme l'est le sens même à donner au mot. L'histoire du terme et le repérage de ses occurrences, nombreuses, dans les textes médiévaux au-delà de la chanson de geste confirment la complexité de cette forme de

\footnotetext{
${ }^{62}$ Les promesses, «abstraction faite qu'elles sont vraies ou fausses, font quelque chose (et ne se contentent pas de le dire) » (J. L. Austin, Quand dire, c'est faire, p. 37 sq.).

${ }^{63}$ Voir infra. O. Ducrot rappelle que par ce type d'acte de langage, « le locuteur, en l'accomplissant, se rend responsable de la vérité de ce qu'il affirme, accepte d'être mis personnellement en en cause si ce qu'il a asserté se révèle faux » («Illocutoire et performatif », Linguistique et sémiologie, t. 4, Paris, 1977, p. 29).

${ }^{64}$ On retrouve un thème déjà bien analysé par la critique : P. Ménard, dans Le rire et le sourire, montre que le $g a b$-« plaisanterie » l'emporte largement sur le gab-« raillerie » dans les textes littéraires. Voir aussi le débat entre J. L. Grigsby et G. S. Burgess.

${ }^{65}$ Je renvoie à la conclusion pour cette « compromission » divine dans un miracle de minimis qui évoque la production hagiographique de Bernard d'Angers, ou celle de Fleury.
} 
parole. John L. Grigsby ${ }^{66}$ l'a mise en rapport avec ce qu'il appelle des «fanfaronnades » d'après boire entre guerriers, repérables dans un grand nombre d'aires culturelles et linguistiques, depuis les Germains de Tacite jusqu'aux Lombards de Paul Diacre et aux sagas médiévales ${ }^{67}$. Il a souligné l'importance cruciale d'une véritable pratique sociale, décrite certes comme parole querelleuse (chez Tacite) ou injurieuse d'après boire (chez Paul Diacre ${ }^{68}$ ), mais qui relèverait aussi du rite norrois du mannjafnadr, soit « comparaison des hommes », cette fois dans et par la collectivité, en l'absence de l'ennemi visé par les paroles. Le gab médiéval, étymologiquement, pourrait ainsi bien dériver de l'anglo-saxon gilp, « vœu », que le jeune homme prononce en vidant sa coupe pour devenir l'héritier de son père chez les Vikings (lors de la heitstrengingar, "cérémonie des vœux »). Et le problème de l'extraordinaire perméabilité de cette parole aux deux catégories du jeu (non opératoire) et du serment ou de l'ordre (qui le lient à une réalisation effective) reste entier en ces traditions : John L. Grigsby rapporte le cas d'une saga (la Jomsvikinga saga) dans laquelle le roi Swein pousse ses guerriers aux «vœux» traditionnels en leur faisant boire sa plus forte bière. Ces «vœux» parfaitement énormes (conquérir la Norvège, en l'occurrence) conduisent en ce cas à l'action guerrière effective, puis causent la mort (dans la guerre) de tous les participants au «concours », sauf un... On retrouve cette même ambiguïté constitutive de la parole gabeuse dans les difficultés de traduction rencontrées par... les traducteurs norrois (pour qui gabba signifierait «ouvrir grand la bouche, railler ») : P. Aebischer montre bien que face à notre texte, les traducteurs ont préféré parfois insister sur l'accomplissement de la parole-jeu en utilisant ithrot, «exploit, exercice », ou «haut fait non imaginaire ${ }^{69}$, ce qui est une interprétation (et une traduction) parfaite de la force perlocutoire du $g a b$.

$\mathrm{Si}$ nous revenons maintenant aux cotextes linguistiques qui accueillent le terme en ancien français au XII ${ }^{\mathrm{e}}$ siècle $^{70}$, il faut noter que certaines occurrences du substantif, symptomatiquement, se présentent dans des propositions interrogatives (type est ce gas ?), ou dans la formule tenir a gab/gas («considérer comme un gab»). Les difficultés d'interprétation et les enjeux incontrôlables du gab, qui certes peut parfois s'interpréter en raison de sa faible charge perlocutoire comme une «plaisanterie», sont donc manifestement pris en compte dans les récits, et pas seulement dans le Voyage. C'est ce que montre une scène du Conte du Graal de Chrétien de Troyes relevée par John L. Grigsby: le sénéchal Keu, que le romancier champenois construit tout au long de son

66 «Le gab dans le roman arthurien français ».

${ }^{67}$ Mais on pourrait aussi bien évoquer la cantoria nordestine du sertaõ brésilien, reconfigurée en « duels de tchatche » par C. Sicre et J.-M. Enjalbert, et qui ressemble aussi à s'y méprendre à une telle pratique, si l'on prendre garde là encore aux différences irréductibles de contexte.

${ }^{68}$ Mais aussi, comme l'a montré F. Mora, dans une épopée comme le Waltharius : outre son éditiontraduction du texte (La Chanson de Walter, éditée et traduite par S. Albert, S. Menegaldo, F. Mora, Grenoble, 2008), on peut consulter L'Enéide médiévale et la chanson de geste, Paris, 1994, p. 170-171.

69 Les versions norroises, surtout p. 42 sq. Voir l'expression segia sina ithrottir, « raconter ses accomplissements ». Inversement, comme le montreraient les traducteurs de la Karlamagnus saga (on en trouvera la traduction par D. Lacroix dans La saga de Charlemagne, traduction française des dix branches de la Karlamagnus saga norroise, traduction, notice, notes et index par D. Lacroix, Paris, 2000), le mot aevintyr (< français aventure), qui signifierait «conte, histoire », est parfois préférable : cela signifierait peut-être en ce cas que le gab reste un jeu privé.

${ }^{70}$ Cfr à ce sujet P. Ménard, Le Rire et le sourire dans le roman courtois en France au Moyen Age (11501250), Genève, 1969, p. 19 sq., qui évoque cette même partition entre le gab appartenant à la catégorie de la «gâté » (dont relèveraient les gabs du Voyage, première manifestation dans la littérature épique du gab« vantardise » tandis que la première occurrence du terme, dans la Chanson de Roland, serait du ressort de la « plaisanterie »), et le gab appartenant à la catégorie de l' «ironie » (dont ferait partie le gab-« raillerie ») : selon l'analyse de P. Ménard, « le sens de 'railler' est le plus répandu de tous les sens de gaber» (p. 25) mais « quant à la valeur de 'vantardise', elle est nettement plus rare » (p. 28). 
œuvre comme un personnage de gabeur, ordonne au jeune Perceval le nice (arrivé à la cour pour s'y faire adouber) de prendre lui-même ses armes/son armure sur le redoutable Chevalier Vermeil car, dit-il, elles lui appartiennent («Amis, vos avez droit./ Alez les prandrë orandroit, les armes, / car eles sont voz », soit «Ami, vous y avez droit. Allez les prendre sur-le-champ, ces armes, car elles sont à vous », v. 1001-1003). Le roi Arthur le tance vertement pour ce gab qui revient, dit-il, à «prometre sanz doner» (v.1016) ${ }^{71}$. Or Perceval, chevalier élu de Dieu s'il en est, accomplit effectivement l'ordre de Keu et donc, réalise cette promesse : il tue le Chevalier Vermeil et s'empare de ses armes, de son armure. Plus tard dans le récit, rétrospectivement interrogé sur cette scène, le roi Arthur explique (en citant le gab au discours direct: «Frere, li rois vos done/ les armes et vos abandone », «Ami, le roi vous donne et vous abandonne ces armes/ cette armure », v. 4009-4010) la façon dont Perceval a interprété ce $g a b:$ «Cil cuida que voir li deïst » (« Il s'imagina qu'il lui disait quelque chose de vrai », v.4101). Le romancier, en soulignant l'équivalence entre le gab et la promesse, insiste sur la manière dont, encore une fois, le $g a b$ échappe contre toute attente à son énonciateur pour s'accomplir dans l'histoire et désigner ainsi, mais nous y reviendrons, celui qui est peut-être appelé à posséder de plus hautes valeurs (mais le roman est inachevé). C'est sur la nature exacte du pouvoir du gab et aussi de son accomplissement que nous allons à présent nous interroger.

ANALYSE NARRATIVE : LES GABS ET SERMENTS DANS LE SYSTEME CAUSAL DU RECIT ET DE L'HISTOIRE

Le texte du Voyage de Charlemagne prend grand soin de préciser l'enjeu du récit en mettant en lumière une chaîne de causalité dans laquelle les gabs prennent toute leur place, comme autant d'actes fondateurs directs dans le déroulement du récit et de l'Histoire de la chrétienté aussi bien : si nous résumons abruptement le texte, nous avons bien affaire à une parole qui est, sans solution de continuité, rien moins que la cause de la préservation ou de l'organisation de l'espace chrétien au sein de l'empire de Charlemagne et ce, en l'absence d'actions guerrières (la «bataille campel ») comme le texte le souligne à deux moments fondamentaux : en ouverture de la série des gabs (le désir de la conquête ouvre l'échange), et à l'extrême fin du texte (c'est en l'absence de bataille que la conquête a eu lieu). Sur le plan narratif, la situation finale permet à Charlemagne, «aimé de Dieu », de contenir tout l'espace chrétien sous sa puissance, de devenir le défenseur, par la parole et par les armes, de l'Eglise, et de transférer de Jérusalem à l'Occident des reliques qui récapitulent toute la temporalité de l'histoire de l'Eglise depuis l'Incarnation ${ }^{72}$.

Cette structuration narrative de la causalité épique que nous allons à présent interroger n'est pas propre au Voyage : nous pourrions aussi bien résumer de la même manière, tout aussi abruptement, certains textes du cycle de Guillaume d'Orange ou la Chanson de Roland, parce que les gabs, mais aussi d'autres formules, très proches de la parole gabeuse, y abondent, pourvues de la même fonction causale dans le récit et dans l'Histoire chrétienne aussi bien. Elles fondent en effet l'action d'un héros qui, invariablement, «molt essauça sainte crestïente $z^{73}$ ». Plus précisément, les formules en question, qui ont fait l'objet des travaux décisifs de Christiane Marchello-Nizia ${ }^{74}$, contiennent un $s i$, adverbe très important dans le développement du système de l'assertion et de la véridiction en ancien français au XII ${ }^{\mathrm{e}}$ siècle (et que déjà, les Serments de Strasbourg utilisaient). Par certaines de

\footnotetext{
${ }^{71}$ Le Conte du Graal, éd. F. Lecoy, Paris, 1975, t. I.

${ }^{72}$ Cfr C. Rossi, «Le Voyage de Charlemagne ».

${ }^{73}$ Charroi de Nîmes, éd. et trad. C. Lachet, Paris, 1999, v.12.

${ }^{74}$ Dire le vrai.
} 
ces formules en si, et comme l'explique Christiane Marchello-Nizia dans ses analyses, le personnage jure d'accomplir une prouesse intenable (prouesse qui s'accomplit, là encore, en faveur de l'espace de la chrétienté), et la met en relation pour l'asserter avec une épreuve pénible ou une privation très dure exercée à l'encontre de son corps, qui peut programmer sa mort. Les locuteurs glosent ces formules par le vocabulaire du serment : plevir, jurer, serement. Le serment est donc prêté sur le corps du locuteur, qui fonde ainsi le dire vrai sans aucune référence directe au divin, comme si son propre sacrifice, suggère Christiane Marchello-Nizia, suffisait à figurer cette dimension spirituelle. Cette formule en si rejoint aussi le gab sur ce point, parce que comme lui, il engage le corps, son exaltation et aussi sa mutilation/humiliation. Guillaume projette par exemple la prise d'Orange en utilisant une telle formule :

Ne mengerai de pain fet de farine...

S'avrai veü com Orenge est assise (La prise d'Orange, v.285-287) ${ }^{75}$

(Je ne mangerai plus de pain fait de farine,

Avant d'avoir vu comment Orange est située...)

Ces formules en si (qui utilisent le futur) permettent au locuteur de marquer son statut juridiquement légitime d'asserteur et sont très proches du gab par leur caractère intenable, sans en revêtir forcément la nature joueuse et railleuse.

Comme l'a démontré la thèse de Christiane Marchello-Nizia, le mot si entre dans d'autres formules de véridiction, qui réfèrent cette fois directement à l'amor Deu, par exemple la formule en si m'aït Dex ${ }^{76}$ :

Si m'aït Deus qui ne menti,

Jeo nel lerroie por murir

Que jeo ne l'auge ja ferir (Gormont et Isembart, v. 208-210) ${ }^{77}$

La paraphrase proposée (« [que Dieu, qui ne fait jamais défaut, m'assiste] est un énoncé aussi vrai que [je ne renoncerais pas à mon projet d'aller le combattre, dussé-je en mourir] ») démontre cette volonté de mettre en scène une parole efficace à la source des actes héroïques de la chanson.

Par le gab, comme par ces différentes formules de jurement ou par d'autres formules encore $^{78}$, le locuteur épique trace pour lui-même (et au-delà de lui : il n'est qu'un maillon

${ }^{75}$ Ed. C. Régnier, Paris, 1986 ( $7^{\text {ème }}$ éd.).

${ }^{76}$ Bien moins caractéristique du récit épique puisqu'on la trouve à foison dans les romans courtois.

${ }^{77}$ Ed. A. Bayot, Paris, 1931.

${ }^{78}$ A noter que la narration épique, de fait, est parfaitement bien représentée par une autre formule en $s i$ que présente le livre de C. Marchello-Nizia : dans le cours du récit épique ou courtois, on trouve ainsi la formule ainc ne fina si..., suivie d'un verbe d'action, qui révèlerait, toujours grâce à l'emploi de $s i$, la présence d'un acte d'assertion y compris dans les passages narratifs et qui serait la marque d'un discours indirect libre : à ce moment-là, le tour en si est « le marqueur d'une volonté : il est référé à un personnage ramassant ses forces sans relâche (ainz ne fina) pour parvenir au but qu'il s'est fixé » et soulignant ainsi avec extase l'aboutissement du vœu initial. 
de la chaîne des causes qui s'origine en Dieu) un destin inéluctable et vrai qui concerne le destin de la chrétienté (sa préservation ou son exaltation) et dont sa parole outrée est la révélation mais aussi la cause directe, quoi que partielle :

Aventure, le maitre mot de[s romans arthuriens en prose] est quasiment absent des chansons de geste ; les héros épiques décident de leur sort, et la présence du si d'assertion dans leurs formules de jurement ou de promesse est la marque qu'ils entendent gouverner leur destin : $s i$ est la trace de leur effort pour transformer la parole prophétique en acte, en réalité. Si est l'interstice, et la jonction discursive, entre la parole de désir et le fait accompli ${ }^{79}$.

Ainsi, le texte épique rêve, dans ces formules-jurement en si et ces gabs, une parole inéluctablement efficace en ce qu'elle garantit et réalise dès sa profération la prouesse épique la plus haute (sur le plan individuel) et la plus indéniablement nécessaire à la chrétienté (sur le plan collectif).

Nous pouvons aller plus loin dans cette analyse structurale de la causalité qui se fonde sur l'efficacité de la parole gabeuse et promissoire : les paroles du héros épique, dessinant son destin inéluctable et celui de la communauté chrétienne, sont en effet autant de développements analeptiques et proleptiques ${ }^{80}$. Autrement dit, la construction de la causalité et de la finalité épiques se trouve concentrée en ces «anachronies » et échappe complètement au niveau horizontal, celui de la « chaîne sémantique » du récit en son cours temporel et causal ${ }^{81}$. Or, il se trouve que l'une des caractéristiques de la chanson de geste $\mathrm{du} \mathrm{XII}^{\mathrm{e}}$ siècle est bien cette «répugnance » à déployer les circonstances de l'action ${ }^{82}$, notamment les liens de cause et de conséquence. Cela donne à la chanson de geste, parfois, un caractère hiératique et tragique peu propice aux justifications, comme l'avait bien analysé E. Auerbach :

Le poète n'explique rien; et pourtant ce qui a lieu est exprimé avec une vigueur paratactique qui signifie que tous les événements doivent se produire tels qu'ils se produisent, qu'ils ne sauraient être différents, et qu'il n'est nul besoin, pour les expliquer, de particules de liaison $^{83}$.

Dans le Voyage, et si l'on structure narrativement la chaîne des causalités, la reine, puis Charlemagne tentent de convaincre leurs interlocuteurs de la gratuité de leurs paroles gabeuses: cela se passe au niveau horizontal du récit, dans la «succession des événements $^{84} \gg$. Mais parallèlement à ce premier niveau, se déploie le niveau de la narration qui réalise, quant à elle, la liaison entre les épisodes : c'est parce que les gabs ont été proférés que s'établit la supériorité, garantie par Dieu «pur amur Carlemaigne » («par l'amour de Charlemagne », v.791), de l'Eglise occidentale au moyen de la translation des

${ }^{79}$ C. Marchello-Nizia, Dire le vrai, p. 64.

${ }^{80}$ Donc autant d' «anachronies », selon la terminologie de G. Genette (cfr en particulier Figures III, Paris, 1972, p. 122 sq.).

${ }^{81}$ D. Boutet, La Chanson de geste, Paris, 1993, particulièrement «La structuration du récit », Deuxième partie, 6, p. 159 sq. : p. 160.

${ }^{82}$ E. Auerbach évoque le «schématisme de la représentation » de la Chanson de Roland (dans Mimèsis. La représentation de la réalité dans la littérature occidentale, Paris, 1968 (trad. frçse), p. 111).

${ }^{83}$ Ibidem, p. 111.

${ }^{84}$ D. Boutet, La chanson de geste, p. 160. 
reliques puis de la translation des valeurs de chevalerie depuis l'Orient vers l'Occident, depuis la mythologie d'Hugon à la charrue d'or jusqu'au royaume historique des Francs, voire la fin de l'Histoire chrétienne ${ }^{85}$. On retrouve ici une structure narrative décrite par E. Auerbach justement à propos de l'écriture chrétienne de l'histoire et qui suppose :

... une relation entre deux événements qui ne sont reliés ni temporellement, ni causalement, une relation qu'il est impossible de postuler raisonnablement dans la dimension horizontale. [...] On peut seulement établir ce rapport en rattachant les deux événements verticalement à la providence divine, seule capable de tracer le plan d'une telle histoire et de fournir la clé de sa compréhension. La liaison horizontale, c'est-à-dire temporelle et causale, des événements se dissout, le hic et nunc n'est plus un simple élément d'un processus terrestre, mais en même temps quelque chose qui a toujours été et qui s'accomplit dans le futur; au fond, devant le regard de Dieu, il est quelque chose d'éternel, d'omnitemporel, qui s'est déjà réalisé fragmentairement dans le domaine des événements terrestres ${ }^{86}$.

Horizontalement, sur le plan du récit, le lien causal entre les gabs et l'accomplissement de l'Histoire est flouté : il n'y a pas d'explications de la part du narrateur et sur le plan linguistique, comme on l'a vu, les choses sont peu contrôlables. Ce lien causal est d'ailleurs inacceptable dans l'ordre de la véridiction (le gab ne saurait être vrai, comme le reconnaissent les locuteurs eux-mêmes) et sur le plan éthique (comment lier une si haute dimension à un tel jeu de paroles et à de telles «intentions » ${ }^{87}$ ?). De même, les « fragments » dispersés de récit que sont les scènes d'accomplissement des gabs après leur profération (la nuit d'amour d'Olivier, le lancer de Guillaume et l'inondation provoquée par Bernard, ou plus largement, la quête vaniteuse de Charlemagne) n'ont sur le plan horizontal (véridiction, éthique et causalité) aucun lien avec l'accomplissement de l'Histoire. Pourtant, ce lien causal horizontal est tissé par la chanson dès lors que l'amur Deu est invoqué par le narrateur et par le locuteur du gab ou du serment ${ }^{88}$ : le gab et le serment épique s'accomplissent en actes qualifiés de miracles et actualisant la vertu de Dieu, ce qui établit un lien cohérent entre le gab, le serment et le dessein divin, alors même que toute causalité horizontale est effacée :

Ne fu mie par force, mès par la Deu vertud (v.751)

Deus i fist tel miracle, li glorius del cel (v.774)

Deus i fist grant vertut pur amur Carlemaigne (v.791)

${ }^{85}$ E. Köhler, L'aventure chevaleresque : Idéal et réalité dans le roman courtois, Paris, 1974 (trad. frçse), surtout «Chevalerie-Clergie : double vocation et conscience historique de la chevalerie courtoise », p. 4476 ; D. Boutet, Formes littéraires et conscience historique. Aux origines de la littérature française (11001250), Paris, 1999.

${ }^{86}$ E. Auerbach, Mimésis, p. 84-85 et sur ce même sujet, Figura. La loi juive et la promesse chrétienne, Paris, 2003 (trad. frçse). On peut évoquer ici Grégoire de Tours, en particulier, parfaite illustration de cette écriture spécifique de l'Histoire, empruntée à la Bible.

${ }^{87}$ Nous sommes ramenés ici aux débats sur les conditions de réalisation de la parole, qui se pose avec acuité dans l'Eglise grégorienne à propos du rôle joué par le ministre chargé du sacrement notamment : cfr la note 12 et les travaux d'I. Rosier-Catach.

88 « amur » reconnu aussi par le « destinataire » de l'effet final du gab, Hugon. 
La vertu qui donne sa valeur spirituelle à la « force » des héros n'est plus médiatisée par la parole des clercs, dont l'acte du chevalier épique fait ici l'économie : Dieu intervient dès lors qu'il est appelé à le faire. Cet appel à Dieu est d'ailleurs direct dans les serments et indirect dans les gabs, qui par définition ne sont pas destinés à être réalisés selon l'énonciateur. Reste que les paroles des chevaliers et du roi font, ici et maintenant, l'Histoire de la chrétienté en lieu et place des paroles des clercs et de leurs sacrements, et sans même utiliser leurs armes si l'on prend le cas du Voyage de Charlemagne. Dans le cas des autres chansons, la fonction guerrière devient quoi qu'il en soit autonome sur le terrain de l'Histoire parce qu'elle est incarnée au préalable par la parole même du guerrier, qui la profère rituellement et appelle ainsi sur lui, sans intermédiaire et sans une autre parole, la vertu de Dieu. Les chansons conteraient donc aussi l'histoire d'un verbe irrévocable, contraignant, efficace, qui trace comme à rebours de son contenu un destin à la chrétienté et contribue à l'organiser en conférant aux héros guerriers un pouvoir de nature finalement spirituelle sur le cours de l'Histoire chrétienne.

FONCTION SOCIO-HISTORIQUE DU RECIT : UNE PAROLE LAÏQUE EFFICACE ET DECLERICALISEE

Le caractère efficace du gab et du serment, dans lesquels s'originent les causes de l'Histoire chrétienne, repose non seulement sur un rapport ambigu au système de la véridiction mais aussi, pour le moins, sur un rapport ambigu aux valeurs dominantes du discours ecclésiastique. Nous voudrions revenir sur ce point en posant brièvement la question si discutée de la fonction socio-historique du récit littéraire.

C'est sans nul doute le gab qui convoque avec le plus d'éclat toute une série de traits évoquant la nébuleuse des péchés de la langue dûment évoqués dans les discours ecclésiastiques, si divers soient-ils au XII ${ }^{\mathrm{e}}$ siècle ${ }^{89}$, qui font face au texte dit littéraire : le gab du Voyage de Charlemagne est proféré dans une communauté de riches laïcs réunis par le péché de bouche en une scène où règnent pêle-mêle intempérance, ivresse, luxure, gourmandise par afflux de vins et de mets recherchés. Se surajoutent à ces traits négatifs la colère, la jalousie et le désir de vengeance et de possession : tout part d'un gab de femme et d'une vantardise de Charlemagne... et se poursuit par le désir âpre de posséder les richesses de la ville orientale. Le gab comprend donc : une amplification extraordinaire des vertus que chacun se prête à soi-même, dans laquelle on reconnaît la jactance, l'orgueil, la superbe du riche ; une injure ou une diffamation; un rapport au mensonge, à la parole inutile, cupide, excessive et obscène, imprudente. De son côté, la formule du serment épique en $s i$, par exemple la formule sacrificielle de Guillaume projetant de prendre Orange dans la Prise d'Orange, est elle aussi motivée par un désir charnel : Guillaume est en fait enflammé au moment où il parle de conquérir la ville sarrasine par son amour de loin pour la reine Guibourc et pour son «beau corps », ainsi que pour les richesses de la ville $^{90} \ldots$ Plus largement, les motivations initiales de Guillaume d'Orange relèvent souvent d'abord de la cupiditas: désir de richesse, envie, orgueil lancent la narration dans le Charroi de Nîmes... et le départ du héros pour l'Espagne.

Nous pouvons ainsi résumer, cette fois sur le plan éthique, les composantes du texte : la chanson de geste propose la réalisation effective dans l'Histoire (dont elle devient la cause,

\footnotetext{
${ }^{89}$ C. Casagrande, S. Vecchio, Les péchés de la langue, que nous suivons dans notre développement.

90 N. Andrieux-Reix, «Une ville devenue désir: la Prise d'Orange et la transformation du motif printanier », dans Mélanges A. Planche, Nice, 1984, t. I, p. 21-32.
} 
après Dieu seulement) de la parole mensongère, obscène et diffamatoire, avec l'aide de Dieu, sans clerc et sans qu'aucun changement d'état soit exigé du laïc... La fonction sociohistorique d'un tel récit, dont on ne connaît ni l'origine, ni l'énonciateur, ni même la date de production, pose problème et des travaux nombreux et érudits ont largement discuté déjà de son sens, de son intention et même du registre utilisé ${ }^{91}$ : satire (cléricale ?) ou parodie de la chanson de geste, de ses formes et de ses valeurs, voire du mythe carolingien, et par là même du groupe des grands laïcs ? Satire (curiale ?) d'un roi, visant Louis VII ou bien Philippe $\mathrm{I}^{\mathrm{er}}$, comme le proposait Reto R. Bezzola ${ }^{92}$ ? Texte écrit à la suite de la canonisation de Charlemagne en $1165^{93}$, exaltant le mythe carolingien et même l'abbé de Saint-Denis au travers des reliques?

On peut peut-être évoquer d'abord, à la suite des nombreux érudits qui ont travaillé sur la question, la question des textes latins qui reprennent ce motif du voyage de Charlemagne à Jérusalem et Constantinople ${ }^{94}$. La Descriptio qualiter Karolus magnus clavum et coronam Domini a Constantinopoli Aquisgrani attulerit, qualiterque Karolus Calvus haec ad Sanctum Dionysium retulerit, ou Iter Hierosolimitanum est par exemple particulièrement intéressante pour notre propos, parce qu'elle permet de bien cerner le discours qui est tenu respectivement sur l'empereur carolingien et sur les clercs, en contexte réformateur: en ce texte, l'activation du motif du voyage vers l'Orient en quête des fameuses reliques permet la mise en scène d'un personnage de souverain présenté moins comme l'auxiliaire de Dieu que comme l'auxiliaire de l'Eglise. Charles y demande en effet à l'archevêque Turpin d'élucider les lettres que lui font parvenir l'empereur oriental et le patriarche de Jérusalem aux abois... L'archevêque Ebroïn (et non plus seulement l'épique Turpin, présent seulement à Aix-la-Chapelle) l'accompagne et le pape Léon intervient, plutôt qu'un «patriarche de Jérusalem » qui évite bien commodément d'évoquer, dans la chanson, le pape de Rome et la hiérarchie ecclésiale. Les cérémonies du texte latin sont organisées par prescription ecclésiastique, une fois établie la fête solennelle des reliques par l'empereur. Autrement dit, le voyage à Jérusalem et à Constantinople illustrerait en ce texte un usage auxiliarisé des armes et la responsabilité d'une Eglise institutionnalisée et hiérarchique dans l'Histoire : il ne donne pas au souverain laïc un quelconque pouvoir sur les signes spirituels. Tout cela n'est pas étonnant en contexte : la période n'est pas à l'exaltation du pouvoir spirituel autonome du souverain... Au fond, dans des textes comme la Descriptio ou encore dans toute l'historiographie fabriquée à Saint-Denis ou dans les vitae réformatrices plus largement, c'est l'auxiliarité du souverain ou du grand laïc en tant que «bras armé » de l'Eglise institutionnelle qui est mise en scène. Ils ne sont en rien la cause de l'histoire : doit-on rappeler quel rôle de contre-modèle joue Charlemagne dans le texte choisi à Saint-Denis et Saint-Germain-des-Prés au XII ${ }^{\mathrm{e}}$ siècle, au moment de la confection des manuscrits recueillant l'histoire des rois des Francs, pour

\footnotetext{
${ }^{91}$ Les débats de la critique à propos du sens même de la chanson sont extrêmement nombreux. Voir un bon état de la question dans A. E. Cobby, Ambivalent Conventions, p. 82 sq.

${ }^{92}$ R. R. Bezzola, Les origines et la formation de la littérature courtoise en Occident, 2 ème partie, Paris, 1960, p. 494 pour Philippe $\mathrm{I}^{\mathrm{er}}$; T. Heinermann, «Zeit und Sinn der Karlsreise », Zeitschrift für romanische Philologie, vol. 56 (1936), p. 500 pour Louis VII et Aliénor.

${ }^{93}$ T. Frings, compte-rendu des Versions norroises de P. Aebischer, Zeitschrift für romanische Philologie, vol. 83 (1957), p. 175-183, dont la théorie est reprise par H.-J. Neuschäfer, « Le Voyage de Charlemagne en Orient als Parodie der Chanson de geste. Untersuchungen zur Epenparodie im Mittelalter (I)», Romanistische Jahrbuch, vol. 10 (1959), p. 101.

94 Le Chronicon de Benoît du Mont-Soracte est le premier texte à aborder la question du voyage de Charlemagne à Jérusalem et Constantinople (il s'y rend pour la gloire de saint André, patron du monastère, à la demande de Benoît). Le premier chroniqueur de la première croisade et la Vie de saint Sardou, de Hugues de Fleury, mentionnent brièvement Charlemagne et son voyage. En ce qui concerne cette mise au point, elle a été considérablement enrichie par les recherches de C. Rossi.
} 
narrer l'histoire de son règne et celui de son fils, soit la Vita Hludovici Pii de 1'Astronome ${ }^{95}$ ? En ce texte, c'est bien le caractère irrémédiablement et spectaculairement épique de l'empereur qui sert d'argument à la dégradation du mythe impérial, tandis que son fils, bien piètre guerrier devenu humble «fils de l'Eglise », subit une constante humiliation qui est aussi la clé d'un « héroïsme » possible du souverain...

C. Rossi explique que le Voyage, texte «humoristique », montre des Francs « aimés de Dieu $»$ et que «c'est Dieu en personne qui est responsable», de sorte que «les accomplissements des gabs sont ainsi de véritables gesta Dei per Francos: le don des reliques par le patriarche est le témoignage de la supériorité spirituelle de Charlemagne, et c'est cette supériorité qui lui vaut le succès dans les épreuves et la soumission d'Hugon ${ }^{96}{ }$. Nous pouvons souscrire en partie à cette analyse, à la condition de rappeler d'abord qu'il y a là une démonstration radicalement audacieuse en contexte grégorien, où il est hors de propos d'envisager un souverain chef d'Eglise, fût-il issu du passé carolingien ${ }^{97}$ et où est posée la question de la responsabilité exclusive du ministre de l'Eglise dans la révélation et la diffusion de la grâce divine, à travers les sacrements notamment. On voit mal, dans le même ordre d'idée, comment on pourrait rapprocher le propos de notre texte de celui des textes latins de la période sans souligner qu'ils représentent le rôle de Charlemagne dans l'acquisition des reliques et la défense de la chrétienté de manière bien différente : dans notre texte, l'empereur et ses pairs sont choisis par Dieu directement et deviennent ceux qui font l'Histoire, après Dieu et parce que Dieu le veut bien ! Le texte du Voyage n'est pas à une audace près pour mener à bien sa construction polémique et en ce sens, on perçoit qu'il aborde la question de la médiation par rapport au divin : il transfigure le temps d'une aventure le roi le plus épique en roi christique ${ }^{98}$ et ses pairs en apôtres, figurant peut-être même le partage des eaux devant le peuple en marche vers la terre promise. Ajoutons que le pèlerinage à Jérusalem (et la «conquête de Constantinople » aussi bien) est narré non pas tant comme parcours de pénitence que comme un moment de glorification et d'exaltation, de mimétisme typologique entre l'empereur et une figure de Christ glorieuse, «fiere ». De plus, et cette notation du texte est fondamentale, la figure du Christ évoquée ici est une figure de Dieu prédicateur : le roi siège dans l'église et sur la chaire depuis laquelle le Christ «chantat la messe » et «si firent les apostle» (v. 115). Le texte prend ici très explicitement le pouvoir sur une des paroles centrales du système ecclésiastique, celle qui a justement pour fonction d'édifier et de convertir le laïc. Ce faisant, il attribue aux personnages laïcs un pouvoir de nature spirituelle qui leur appartient en propre : c'est le roi qui transfère les reliques et les vertus depuis la terre sainte et surtout, qui les intègre à l'autel dionysien et au reste de son royaume où il les répartit, sans que soit présent aucun clerc. Le Voyage, autrement dit, ne reprend pas mais récrit complètement la version ecclésiastique de l'Histoire et de ses causes, les gesta Dei qui depuis Augustin et Orose

\footnotetext{
${ }^{95}$ P. Bourgain, «La protohistoire des Chroniques latines de Saint-Denis (BNF, lat. 5925) », Saint-Denis et la royauté Saint-Denis et la royauté. Etudes offertes à Bernard Guenée, F. Autran, C. Gauvard, J.-M. Moeglin (dir.), Paris, 1999. p. 375-394.

96 «Le Voyage de Charlemagne », p. 651.

97 Cfr D. Iogna-Prat, «La construction biographique du souverain carolingien », A la recherche de légitimités chrétiennes. Représentations de l'espace et du temps dans l'Espagne médiévale (IXe - XIII siècle), P. Henriet dir., Annexes des cahiers de linguistique et de civilisation hispaniques médiévales, vol. 15, Lyon, 2003, p. 197- 224. Repris et remanié dans La Maison Dieu. Une histoire monumentale de l'Eglise au Moyen Age (v. 800-v. 1200), Paris, 2006, p. 119 et suivantes. Voir aussi A. Boureau, «Un obstacle à la sacralité royale en Occident : le principe hiérarchique », La royauté sacrée dans le monde chrétien, A. Boureau et $\mathrm{C}$. S. Ingerflom dir., Paris, 1992, p. 29-37, p. 31.

98 Admirable retournement du scénario adopté par l'Astronome qui, en vrai «moraliste carolingien », avait su ouvrir la voie aux personnages de rois cléricalisés, à la suite de Jonas d'Orléans, mettant fin à une conception bien différente de la souveraineté : cfr D. Iogna-Prat, « La construction biographique ».
} 
jusqu'aux manuscrits de Saint-Denis en particulier, manifestent la loi du genre de l'historia ecclesiastica : c'est l'Eglise (et ses hommes) qui fabriquent l'histoire, non les rois ${ }^{99}$. Notre texte prend le contre-pied d'une telle construction.

On peut douter cependant que les liens tissés avec cette tradition textuelle ecclésiastique contemporaine, évoquée au début de notre analyse, soient de simple parodie ou même d'opposition brutale : dans la chanson en langue romane, la décléricalisation du propos et des structures du récit est spectaculaire, et originale la structure narrative qui consiste à supprimer le motif des armes ou à la soumettre aux gabs et serments des chevaliers euxmêmes pour évoquer la fonction du roi et des grands. Si on la compare aux constructions ecclésiastiques réformatrices (et notamment aux scènes qui confrontent les clercs aux aristocrates dont ils font les bras armés de l'Eglise), voilà une construction hybride qui met en cause de manière au fond très complexe les schémas de hiérarchisation clercs/ laïcs proposées par différents énoncés ecclésiastiques. Les rapports entre les discours ne seraient donc pas tant d'opposition que de recomposition. Essayons d'en récapituler tous les fils : tout d'abord, la chanson de geste emprunte au discours ecclésiastique à la fois le motif d'une fonction guerrière aristocratique et le principe d'une domination exclusive de la dimension spirituelle sur la dimension charnelle (qu'elle ne remet jamais en question, comme le montre Anita Guerreau-Jalabert dans ses recherches ${ }^{100}$ ). Mais elle reprend aussi avec insistance certaines des «tares» du grand aristocrate laïc que dénonce le texte ecclésiastique réformateur: notre texte, comme certaines vitae de la période, représente ainsi pleinement le grand aristocrate laïc en miles-rhinocéros, avec une mobilisation très forte, comme on l'a vu, de ses traits les plus charnels. Le laïc est portraituré en roi ou en chevalier, en puissant avide de rétablir sa puissance, en riche avide de confirmer sa richesse, en orgueilleux, en coléreux et en vantard impudent. Sa parole est jactance, superbe, orgueil, injure, inutilité, mensonge. Et en ce sens, le registre comique, ou ironique selon les critiques, n'est en rien un élément marginal dans cette démonstration : il fait partie déjà des vitae qui ne se privent pas d'exploiter ce registre quand il s'agit d'évoquer les laïcs... ou les clercs déviants. Il est pleinement mobilisé ici dans la configuration du laïc. Mais dans le même temps, tandis qu'il semble acquiescer à ces définitions de la déviance du grand laïc, le texte épique dote certains de ses personnages de traits et d'actions de nature pleinement spirituelle, et d'un lien direct avec le dessein divin, ce que lui refuse le discours ecclésiastique grégorien: Charlemagne ressemble donc, dans la chaire somptueuse de l'église de Jérusalem, au Christ lui-même par son visage fier... Et la parole (même la plus charnelle comme l'est le gab) est réalisée par Dieu, un Dieu que la prière du roi semble contraindre à se compromettre dans les affaires du monde sans l'intermédiaire des clercs. Autrement dit, insistons sur ce point, ce sont des grands laïcs représentés au sein même de leur état conjugal et/ou leurs déviances les plus charnelles (sexualité, avidité...), qui sont des acteurs du grand plan divin sans aucune solution de continuité, par recyclage et conversion de leurs paroles. Ainsi se trouve redéfinie la valeur spirituelle non contestable du personnage du grand aristocrate laïc...

Cette recomposition est propre aux chansons de geste, qui évitent encore d'aborder, à la différences des romans, certains des sujets polémiques à propos du grand laïc. Il semblerait donc que la chanson de geste travaille à dissoudre la «reconnaissance humiliée » d'une

\footnotetext{
${ }^{99}$ Nous nous permettons de renvoyer à notre article : «Exercices de style : amplification de la forme et amplification de la matière dans deux chroniques des rois de France (XIII ${ }^{\mathrm{e}}$ siècle) », Poétique de la chronique. Textes historiographiques et écriture (Péninsule ibérique, France, $X I^{e}$-XIV siècle), A. Arizaleta dir., Toulouse, 2008, p. 153-192.

${ }^{100}$ Cfr notamment «Le temps des créations », où l'on trouvera une bibliographie plus complète. Ces recherches inspirent largement les nôtres.
} 
fonction guerrière qui, dans d'autres énoncés, n'a de valeur que lorsqu'elle est la métaphore vive et dégradée du glaive de la parole cléricale, processus d'englobement dont elle retire l'unique parcelle de pouvoir et de valeur de nature spirituelle à laquelle elle peut avoir accès, et encore, de manière conditionnée. Pour répondre à ces énoncés, la chanson travaille à même la fonction guerrière sans remettre en cause, comme le fera le roman, ce «portrait du grand laïc en miles », ce grimage légué par les textes réformateurs. C'est du sein même de ce motif des armes, et sans doute parce qu'il est l'outil privilégié de cette « reconnaissance humiliée » du laïc dans les énoncés ecclésiastiques réformateurs (comme d'ailleurs la sexualité), que se dessine de manière provocatrice la prise de possession autonome, par le personnage de laïc déguisé en chevalier, d'un pouvoir et de valeurs de nature pleinement spirituelle : morts glorieuses de Roland ou de Vivien ; accomplissement de l'Histoire de la chrétienté par Guillaume ; mais aussi et surtout, de manière peut-être plus ombrée, profération autonome d'une parole efficace qui donne à l'action guerrière des contours transfigurés, au point de la remplacer parfois comme dans le Voyage. Il est temps en ce point de notre enquête de repréciser que l'outrance de la profération (serment parfois prêté sur le corps offert en sacrifice) et du contenu de la parole épique, gab ou serments, a ainsi des limites précises : cette parole s'élabore en rapport avec la seule prouesse physique du chevalier épique, de son corps vaillant, éventuellement du sacrifice de ce corps, à l'exclusion de tout autre type de merveilleux. Ces paroles que les textes qualifient parfois de merveilleuses ${ }^{101}$ parce qu'efficaces et outrées, et les actes merveilleux et hauts qu'elles accomplissent, et qui confinent au miracle, participent ainsi de la construction d'un héroïsme épique voué à arracher la grandeur de l'action guerrière à une auxiliarité seulement terrestre (la force), pour révéler qu'en elle se manifeste la plus haute dimension spirituelle :

Dans la parfaite unité de style de notre épopée, le merveilleux qui surgit encore avec sa valeur étymologique ouvre une faille sur d'autres valeurs que celles de ce monde. La démesure, le dépassement héroïque conduisant à un excès de morts, la rencontre d'un surnaturel qui n'est pas dans l'exact prolongement du geste sublime, préparent la mise en question par la littérature profane, en langue vulgaire, sous le couvert du merveilleux, de la grandeur $^{102}$.

Et cela suffit, pour l'instant et avant les audaces plus grandes des romans, à revendiquer pour l'aristocrate laïc une valeur plus haute que celle qui est dévolue aux rhinocéros imperiti sermone.

\section{CONCLUSIONS PROVISOIRES}

Les chansons de geste proposent bien un acquiescement éclatant à la fonction guerrière à laquelle une partie du discours ecclésiastique réformateur réduit le grand aristocrate laïc pour mieux fonder sur cette fonction la dévaluation du statut et de la valeur du laïc-miles. Mais cet acquiescement semble bien aller de pair avec une tentative de récupération des

\footnotetext{
${ }^{101}$ Il est permis de rappeler que (conformément aux règles de l'amplification épidictique) cet adjectif est aussi bien appliqué en abondance aux actions des personnages épiques qu'aux principaux marqueurs de l'univers épique : chevaux, paysages, armes... et paroles (l'espion qualifie le gab d'Ernalt de « merveillos gab », v.576). Voir J.-R. Valette, La Poétique du merveilleux dans le Lancelot en prose, Paris, 1998.

102 D. Poirion, «Théorie et pratique du style au Moyen Age : le sublime et la merveille », Revue d'histoire littéraire de la France, I (1986), p. 15-22, p. 24.
} 
plus hautes valeurs (de nature inévitablement spirituelles) que cette fonction guerrière leur ôte de fait dans ce discours ecclésiastique. L'appropriation laïque de la guerre implique pour ce faire à la fois une décléricalisation de la parole efficace qui ordonne la guerre et impose son sens, et une refabrication de cette parole qui soit extrêmement précise quant à ses pouvoirs et à la définition de son opérativité : le texte en langue vernaculaire réinvente donc la fonction guerrière à laquelle sont cantonnés les grands laïcs dans le discours réformateur par le moyen de la réinvention d'une parole efficace susceptible de donner au roi et aux chevaliers un rôle véritable, et non plus subalterne, dans l'Histoire. Les milites font ici la chrétienté par leur parole, pas seulement, voire plus du tout, par leurs armes. Il s'agirait ainsi pour la littérature épique d'arracher un héroïsme laïc (au moins rêvé à défaut d'être réalisé) à un usage des armes radicalement inférieur en valeur et largement soumis, dans les autres discours en présence, à l'injonction et au cadrage de la parole ecclésiastique.

Ce faisant, le propos du texte littéraire ne remet pas en cause le principe de la nécessité et de la supériorité du spirituel et c'est en cela qu'il n'y a pas là une simple opposition de discours: les laïcs sont bien, en cette production épique en langue vernaculaire, des personnages positifs parce qu'ils sont du côté d'un spirituel chrétien. Mais ils y ont accès sans médiation cléricale et, du sein même de leur état laïc le plus marqué par la richesse, le désir et la force, leur seule parole spiritualise merveilleusement leurs actions et leur pouvoir sur l'histoire. Ainsi, en opérant ses «scandale(s) d'inversion(s) sociétale(s) » ${ }^{103}$, l'univers épique ne produit pas un renversement complet, mais bien un «déplacement de lignes » à la recherche d'un "écart significatif », comme l'analyse Anita Guerreau-Jalabert à propos des textes arthuriens : cela entraîne une recomposition complète du « surnaturel positif » (le spirituel) au moyen d'éléments qui ne devraient pas en faire partie... Et ce serait l'un des sens de ces textes en langue vernaculaire que de retravailler par tous les moyens un système de représentation qui dévalorise ailleurs systématiquement la classe des laïcs : on le voit avec le motif de la parole laïque, ici complaisamment mise en scène au travers de l'activation de ses traits les plus ouvertement charnels et condamnables, alors que qui la profère se trouve placé en lien causal direct avec le dessein spirituel et une efficacité miraculeuse. La littérature montre ainsi son extraordinaire pouvoir de contournement et de reprise, moins idéologique d'ailleurs qu'utopique au sens ricœurdien du terme : ni saints, ni clercs, ni même laïcs pénitents, pourvus des caractères de la beauté, de la sexualité et du caractère du riche et du puissant, le roi Charles et le chevalier proposent une parole vraie et efficace, cause de l'histoire voulue par Dieu. Et nous n'en sommes pas encore aux gabs tout aussi efficaces et destructeurs, sans doute davantage pour l'Eglise institutionnelle que pour la spiritualité des laïcs, de Renart...

Cette « littérature » marque ainsi que l'une des composantes de son identité est peut-être moins dans l'opposition (et la gratuité) que dans la confrontation aux autres discours, confrontation pour laquelle elle emploie des mécanismes de recyclage et de conciliation de ce qui est présenté comme incompatible dans certains énoncés ecclésiastiques ${ }^{104}$. Un autre exemple de recyclage consiste par exemple en cet apprivoisement du sacré, humilié (volontairement !) jusqu'à la terre au moyen d'une prière, d'un serment ou d'un gab qui

\footnotetext{
103 J. Le Goff, «Les gestes symboliques dans la vie sociale. Les gestes de la vassalité », repris dans Pour un autre Moyen Age. Temps, travail et culture en Occident, Paris, 1977, p. 349-420, p. 382.

${ }^{104}$ Il est bien d'autres points de « recyclage » en ce sens : il faudrait par exemple, et dans le détail même du texte, analyser en ce sens le recours à un personnage de «patriarche » (le mot même est singulier) plutôt qu'à un personnage de pape ; le motif des reliques, etc... mais approfondir aussi bien la réflexion sur la nature de la «causalité » donnée ici à la parole efficace, à la lumière des textes théologiques et des textes juridiques, comme le suggère I. Rosier-Catach, que je remercie bien vivement pour ses remarques stimulantes.
} 
portent des désirs d'accomplissement bien terrestres... Cet apprivoisement n'a en effet rien d'original dans la forme : il est bien présent dans certains miracles de sainte Foy rapportés par Bernard d'Angers. Que l'on se souvienne du miracle accordé au miles qui avait voulu briller aux yeux du monde en empruntant, moyennant une clause désastreuse pour lui, un faucon à un seigneur cupide, et qui perd ce faucon : le miles prononce un vœu et fait une offrande à la sainte, sur son autel et en son église, afin de le retrouver, ce que la sainte accorde sans même demander que les défis s'arrêtent là, contrairement à l'ange de la chanson... Comme l'a montré l'analyse précise de Dominique Barthélemy ${ }^{105}$, il convient de prêter attention à la présence, dans ce texte hagiographique antérieur à nos chansons, d'un développement intéressant de Bernard sur le mot jocum, utilisé par les « habitants de Conques » pour qualifier traditionnellement, dit le narrateur, ce type de miracle. Dominique Barthélemy insiste sur l'originalité du terme : Bernard, dans le terme jocum, soupçonne une interprétation du miracle qu'il récuse et dont il rend responsable le rusticus intellectus des témoins, en proposant de remplacer le terme par l'expression «miracle de minimis ». De fait, à propos d'une histoire qui met en lien l'orgueil du chevalier, sa sottise, la cupidité d'un seigneur, et l'efficacité de la prière, Bernard souligne que le danger le plus grand est le défaut de compréhension à propos de ce qui reste un signe envoyé par Dieu à travers le miracle. Ce signe en effet témoigne, plus que jamais quand il ressemble à un jeu du divin avec des situations relevant de préoccupations seulement mondaines et laïques, de l'opacité des signes divins et de leur extrême complexité. Les jeux doivent permettre d'établir le «principe organisateur du monde : reverentia, péché et malchance », comme l'explique Peter Brown ${ }^{106}$, à propos de désirs, de désillusions, d'accidents qu'on ne comprend pas tout d'abord, mais qui sont imputables finalement aux desseins de Dieu et/ou à une plaisanterie de la sainte omniprésente. Le gab de la littérature épique pourrait bien entrer dans une relation de filiation structurelle et éthique contrariée, certes, mais réelle, avec ce jocum hagiographique. En cela, le narrateur de la chanson appelle peut-être à passer de la lecture-rusticitas à la lecture-reverentia de la parole laïque qu'il réinvente et à laquelle il donne la lourde fonction de causer l'Histoire des hommes ${ }^{107} \ldots$

\footnotetext{
105 «Un jeu de Sainte Foy de Conques : le miracle du faucon retrouvé (Bernard d'Angers, I.23)», dans Retour aux sources. Textes, études et documents d'histoire médiévale offerts à Michel Parisse, Paris, 2004, p. 383-394.

${ }^{106}$ P. P. Brown, «Reliques et statut social au temps de Grégoire de Tours », repris dans La société et le sacré dans l'Antiquité tardive, Paris, 1985 (trad. frçse), p. 185-214, p. 193.

${ }^{107} \mathrm{Je}$ remercie bien vivement M.-P. Halary pour ses relectures attentives et suggestives, ainsi que J.-R. Valette et L. Feller.
} 